\title{
ÜBER FINNLANDS REZENTE UND SUBFOSSILE DIATOMEEN II
}

\author{
Karl Mölder und Risto Tynni \\ Geologische Forschungsanstalt in Finnland, Otaniemi, Finnland
}

\section{ABSTRACT}

The article deals with the distribution and ecology of the Cyclotella, Stepbanodiscus, Coscinodiscus, Actinoptychys, Auliscus, Actinocyclus, Rhizosolenia, Chaetoceros, Attheya and Terpisnoe species known in Finland.

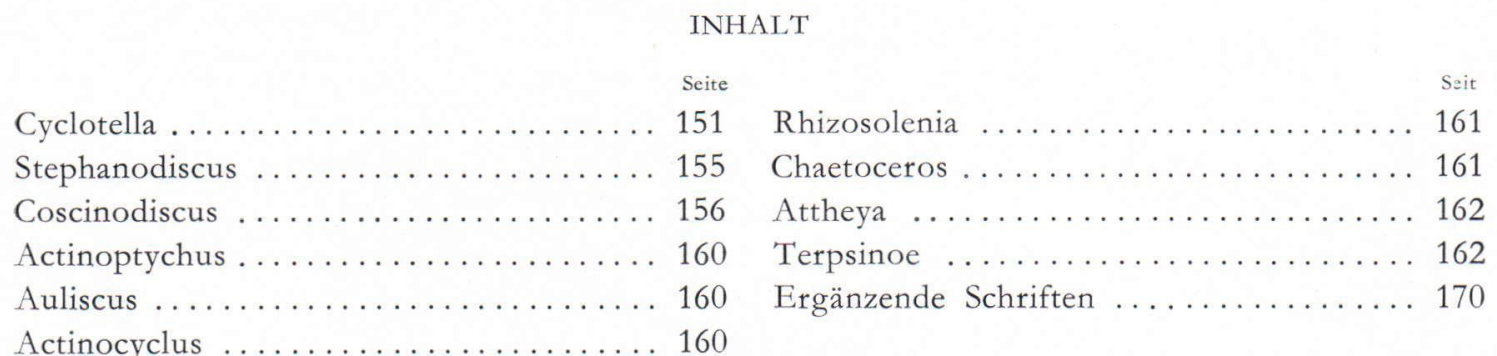

\section{Cyclotella}

Trommel- bis diskusförmige Zellen, die entweder frei oder miteinander zu Kolonien vereinigt leben. Die Valvarfläche ist mehr oder minder gewelt, selten eben und sie ist bei den in Finnland anzutreffenden Arten kreisförmig oder fast kreisförmig (C. iris). In der Randzone radiale Streifung, die sich scharf vom glatten, perforierten oder granulierten Mittelfeld unterscheidet.

Cyclotella antiqua W. Smith.

Synonym Cyclotella operculata var. antiqua Héribaud.

Eine besonders in Lappland in oligotrophen Seen in der Litoralzone selten vorkom.mende Art. (Abb. 1.) Subfossil in spät- und postglazialen Süßwassersedimenten selten.

\section{Cyclotella arentii Kolbe.}

Synonym: Coscinodiscus arentii (Kolbe) Cl.-E.

Nur in Pukkila im Kokkusalampi, einem dystrophen Moorweiher, angetroffen. Abb. 1. Die Art erschien einzeln in einer aus Ufervegetation oder oberfächlichem Bodenschlamm entnommenen Probe.

Cyclotella bodanica Eulenst.

Synonyme: Cyclotella balatonis Pantocsek, Cyclotella comta var. bodanica Grunow.

In einigen Seen gefunden, ist aber offenbar häufiger im pelagischen Plankton oligotropher Seen. Abb. 2. Subfossil in Ancylussee- u.a. Seesedimenten.

Aus dem südkarelischen Weiher Vessillanlampi (Hustedt 1930) und in klarwässerigen Seen in Lappland (Cleve-Euler 1951) ist ausserdem die der Hauptform stark ähnelnde $C . b$. var. lemensis O. Müller dargestellt worden, desgleichen aus Lappland C. b. var. borealis A. Cl. -E. (Cleve- 


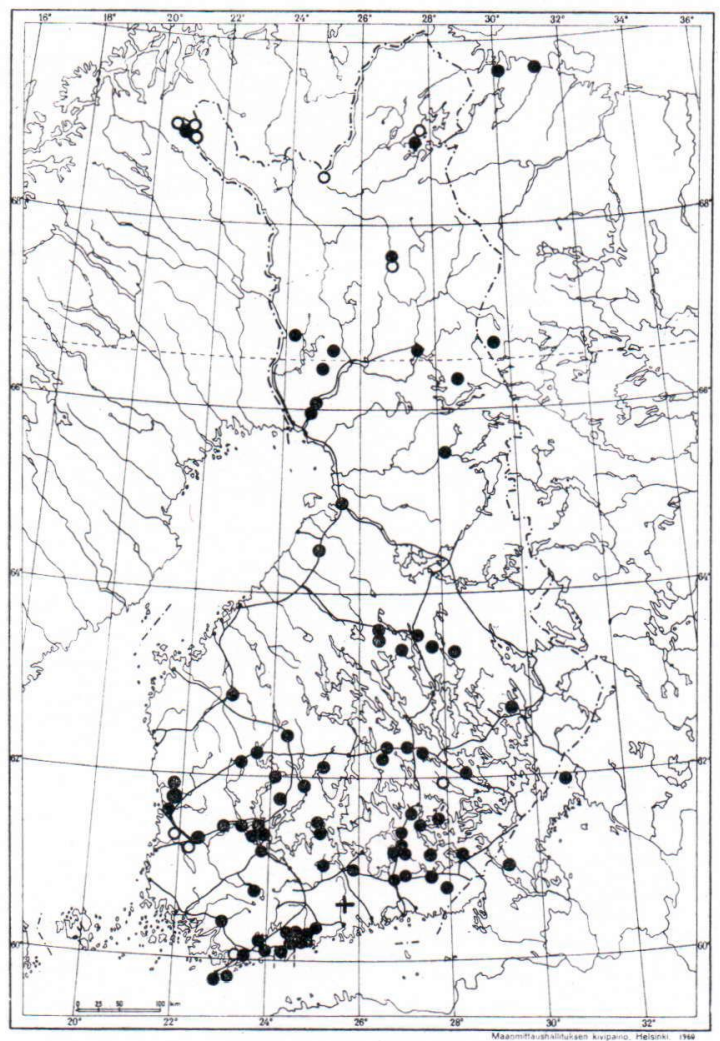

Авв. 1. Rezente Verbreitung von Cyclotella antiqua (Ringe), Cyclotella arentii (Kreuz) und Cyclotella stelligera (Punkte).

Euler 1951). C. b. var. lemensis kommt auch subfossil neben der Hauptart vor, u. a. in den Sedimenten der Päijännesee (Aario 1965).

\section{Cyclotella catenata Brun.}

Synonyme: Melosira catenata Brun, Cyclotella melosiroides von Kreissler

Seltene Art, die nur in Porrassalmi in einem See und in dem eutrophen Tuusulanjärvi angetroffen worden ist. Abb. 2. Subfossil nicht gefunden.

\section{Cyclotella chaetoceras Lemm.}

In Süßwasser pelagisch wachsende Form (Hustedt 1930), nur in dem nordfinnischen Fluss Kaisajoki in Tervola gefunden. Abb. 2.

Cyclotella comta (Ehr.) Kütz.

Synonyme: Discoplea comta Ehr., Actinocyclus belveticus Brun, Cyclotella comta var. lucida Meister, Cyclotella comta

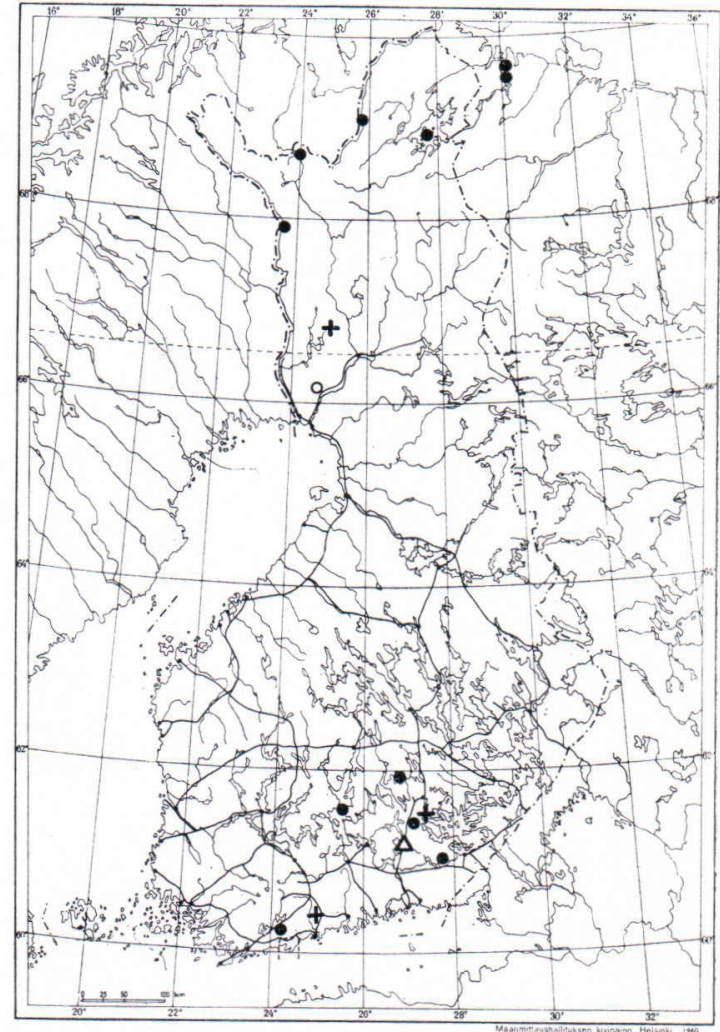

Авв. 2. Rezente Verbreitung von Cyclotella bodanica (Punkte), Cyclotella catenata (Kreuze), Cyclotella chaetoceras (Ring) und Cyclotella comta var. paucipunctata (Dreieck).

var. evidenterpunctata Mayer, Cyclotella comta var. radiosa Grun.

In Finnland die häufigste Cyclotella-Art, die vom Trophiegrad der Gewässer unabhängig, aber wahrscheinlich alkaliphil ist (Meriläinen 1967). Im Plankton von Seen und Flüßen wachsende Art. Abb. 3. Subfossil in Ancylusseeu.a. Süßwassersedimenten. Var. oligactis (Ehr.) Grunow und var. paucipunctata Grun. sind in den Gewässern Finnlands selten. Abb. 3 u. 2.

Var. binota Pant. erw. C. comta var. spectabilis Cleve.

In der Randzone 2-5 flammende Punkte. Die Variation haben Fontell und Lindberg in Finn land (im See Höytiäinen und im Moor Hinder mossen von Karjaa) aufgefunden (Cleve-Euler 1951). 
Cvclotella glomerata Bachmann.

Synonyme: Cyclotella sosialis var. minima Bachm., Cyclotella luzernensis Bachm., Cyclotella bachmanni Meister.

Eine pelagische Form subalpiner Seen ( $\mathrm{Hu}-$ stedt 1930), die in Finnland sehr selten ist. Aus dem Tirvanjärvi in Valkeala, dem Tuusulanjärvi und dem Juusjärvi von Kirkkonummi erhalten. Abb. 4.

\section{Cyclotella iris Brun.}

Eine Form oligotropher kaltwässeriger Seen, die u.a. in den Seen Inarinjärvi, Kilpisjärvi und Säämäjärvi in Aunus vorkommt. Subfossil u.a. in Ancylussee- und Binnensee-Sedimenten angetroffen.

\section{Cyclotella kützingiana Twaites.}

Synonyme: Cyclotella operculta f. rectangulata Kütz., Cyclotella kützingiana var. cataractarum Grun., Cyclotella kuitzingiana var. pelagica Grun., Cyclotella kützingiana var. nobilis A. Cleve

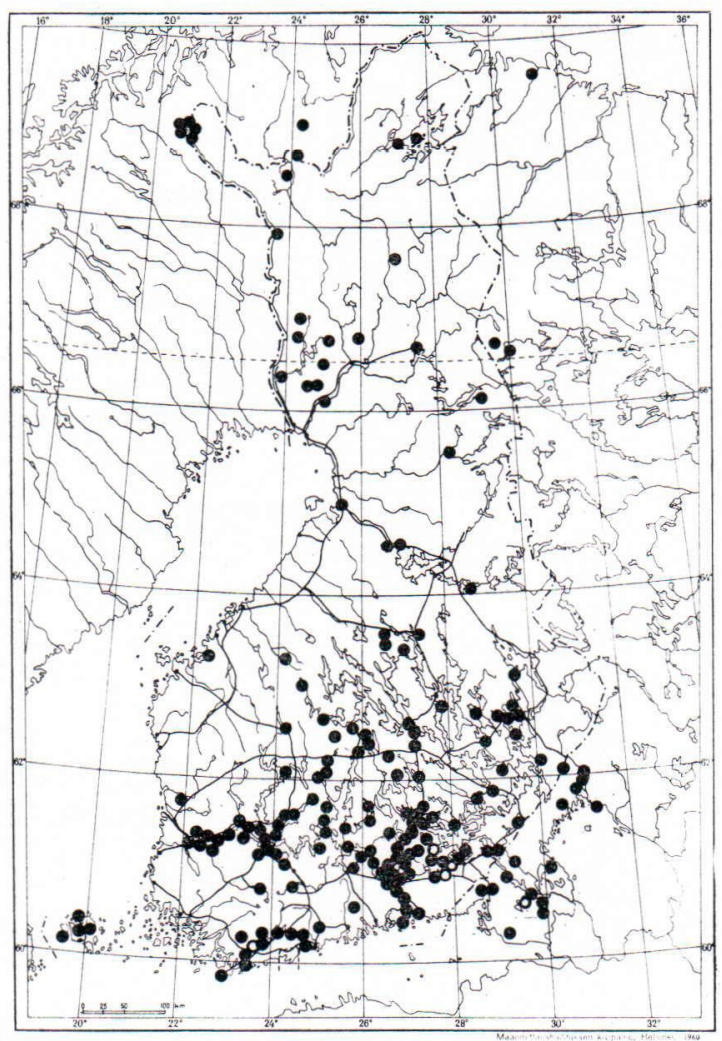

ABB. 3. Rezente Verbreitung von Cyclotella comta (Punkte) und C. c. var. oligactis (Ringe).
Eine besonders in der Litoralzone nährstoffarmer Seen und Flüsse wachsende Form, die selten im Plankton erscheint. Abb. 5. Subfossil in Süßwasser-, u.a. Ancylussee-Sedimenten.

Var. planetophora Fricke.

Die Variation ähnelt sehr $\operatorname{der} C$. ocellata, die von Cleve-Euler (1951) denn auch als Synonym der vorherigen angesehen wird.

Rezent und subfossil selten. Abb. 6.

Var. radiosa Fricke.

Selten wahrgenommene Form, die vielleicht nur mit oligotrophen Gewässern zusammenhängt. Abb. 6.

Var. schumanni Grun.

Seltene Variation, deren Verbreitungsstellen sich auf oligotrophe Gewässer Nordfinnlands konzentrieren. Abb. 5.

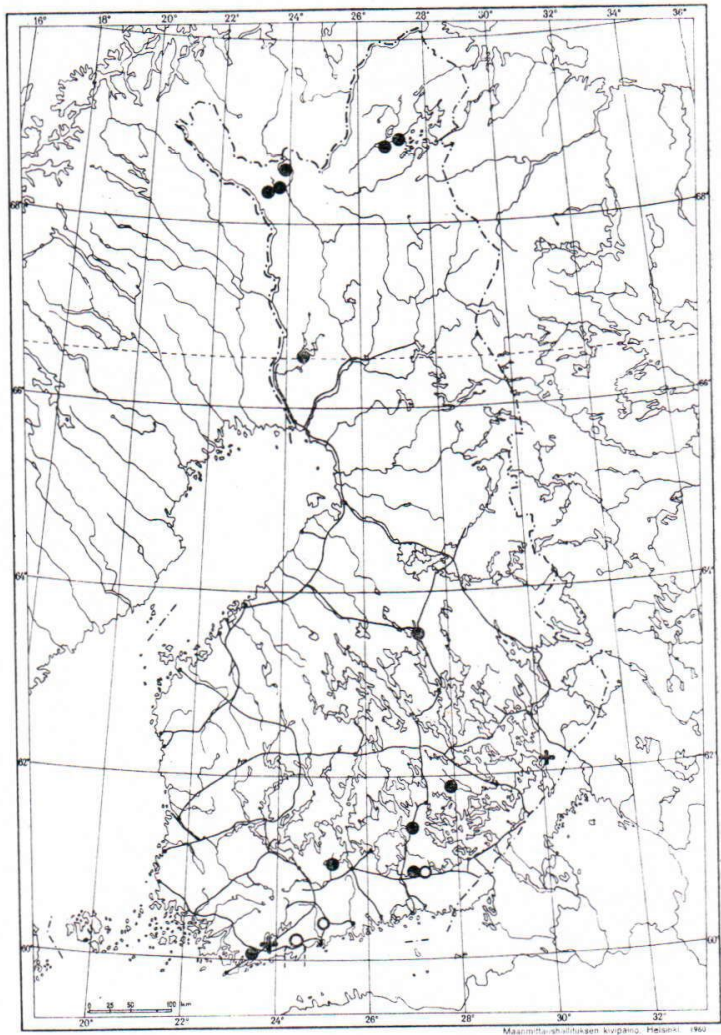

ABB. 4. Rezente Verbreitung von Cyclotella glomerata (Ringe), Cyclotella operculata (Punkte) und Cyclotella striata (Kreuze). 


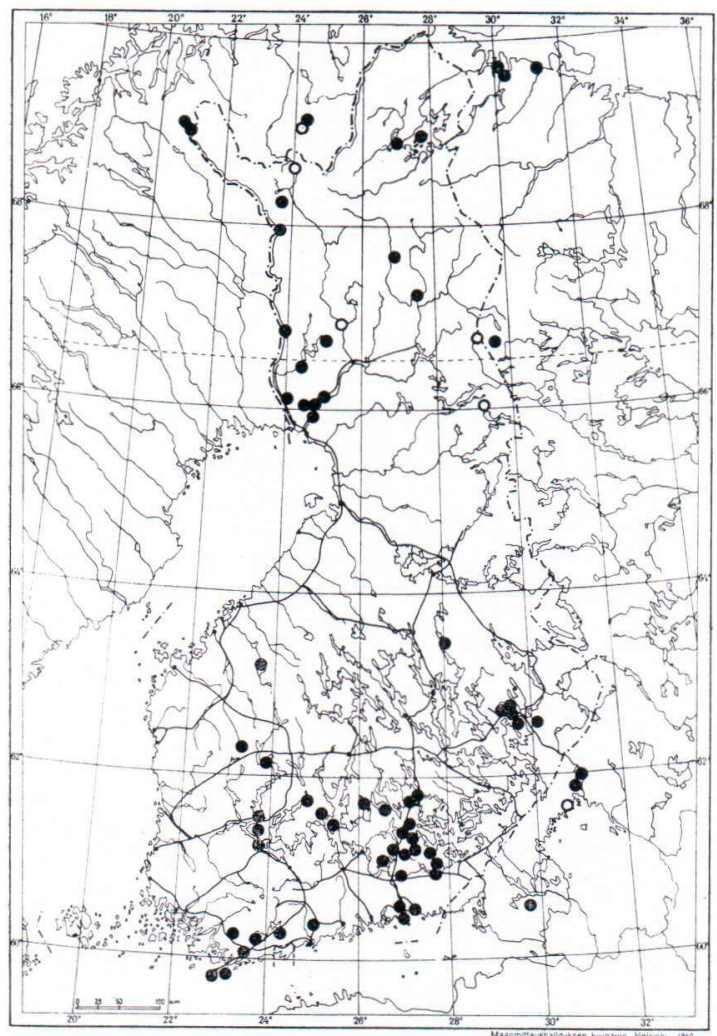

Авв. 5. Rezente Verbreitung von Cyclotella kützingiana (Punkte) und C. k. var. schumanni (Ringe).

Cleve-Euler hat die von uns als Synonyme der Art erachteten Variationen dargestellt: C. K. var. nobilis und C. k. var. catarctarum Grun., erstere aus dem Aapajärvi und letztere aus dem Inarinjärvi.

\section{Cyclotella menegbiniana Kütz.}

Synonyme: Surirella melosiroides Meneghini, Cyclotella melosiroides Meneghini, Cyclotella salina Marsson, Cyclotella meneghiniana var. vogesiaca Grun., Cyclotella rectangula Bréb., Cyclotella meneghiniana var. rectangulata Grun., Cyclotella meneghiniana var. binotata Grun.

In Finnland häufige Art besonders in schwach eutrophen Binnengewässern und in schwach brackwässerigen Litoralzonen der Küste. Abb. 7. Subfossil auch häufig in Sedimenten von Süßund schwachem Brackwasser.

Var. laevissima (van Goor) Hustedt.

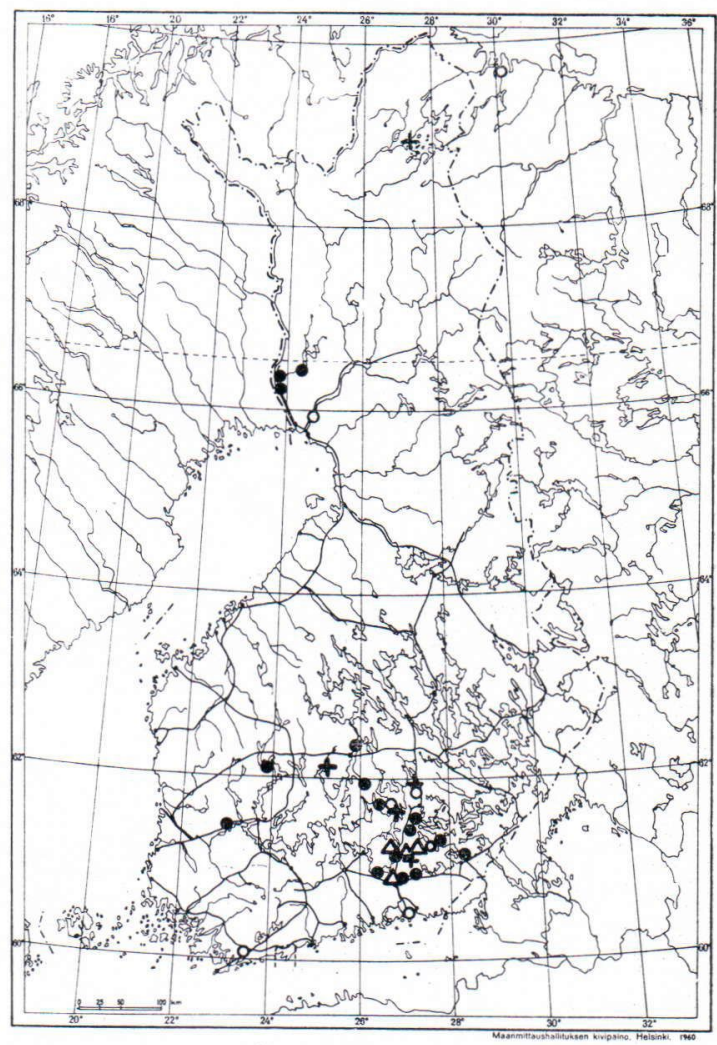

Авв. 6. Rezente Verbreitung von Cyclotella kützingiana var. planetophora (Ringe), C. k. var. radiosa (Kreuze), Cyclotella ocellata (Punkte) und Cyclotella quadriuncta (Dreiecke).

Synonym: Cyclotella menegbiniana var. tenera Kolbe. Für Finnland dargestellt aus verunreinigtem Küstenwasser vor Helsinki als Indikatorart (Välikangas 1926) und von der Insel Lehtisaari (Mölder \& Tynni 1966) Abb. 7.

Cyclotella ocellata Pantocsek.

Synonyme: Cyclotella crucigera Pantocsek, Cyclotella tibetana Hustedt.

In der Litoralzone von Seen angetroffene seltene Art. Abb. 6.

Cyclotella operculata (Ag.) Kütz.

Synonyme: Frustulia operculata Ag., Cymbella operculata Ag., Pyxidicula operculata Ehr.

Seltene Art, die besonders in oligotrophen Seen in der Litoralzone vorkommt. Abb. 4.

Cyclotella quadriiuncta Schröter.

Synonyme: Cyclotella comta var. quadriiuncta Schröter, 
Cyclotella scbröteri Lemmermann.

Die Art ist pelagisch in lockeren Kolonien von 2-8 Individuen in einigen Seen Südfinnlands angetroffen worden. Abb. 6. Einzeln sind die Zellen sicher nicht von $C$. comta zu unterscheiden. Möglicherweise ist die Art besondess in subfossilem Material, wo die Kolonien nicht mehr erscheinen können mit C. comta verwechselt worden.

\section{Cyclotella stelligera Cleve et Grunow.}

Häufige Art in eutrophen bis oligotrophen Gewässern. Abb. 1. Subfossil in SüßwasserSedimenten.

\section{Cyclotella striata (Kütz.) Grun.}

Synonyme: Coscinodiscus striatus Kütz., Cyclotella dallasiana W. Smith, Cyclotella sinensis Ralfs.

Im Küstengebiet in Brackwasser nur selten

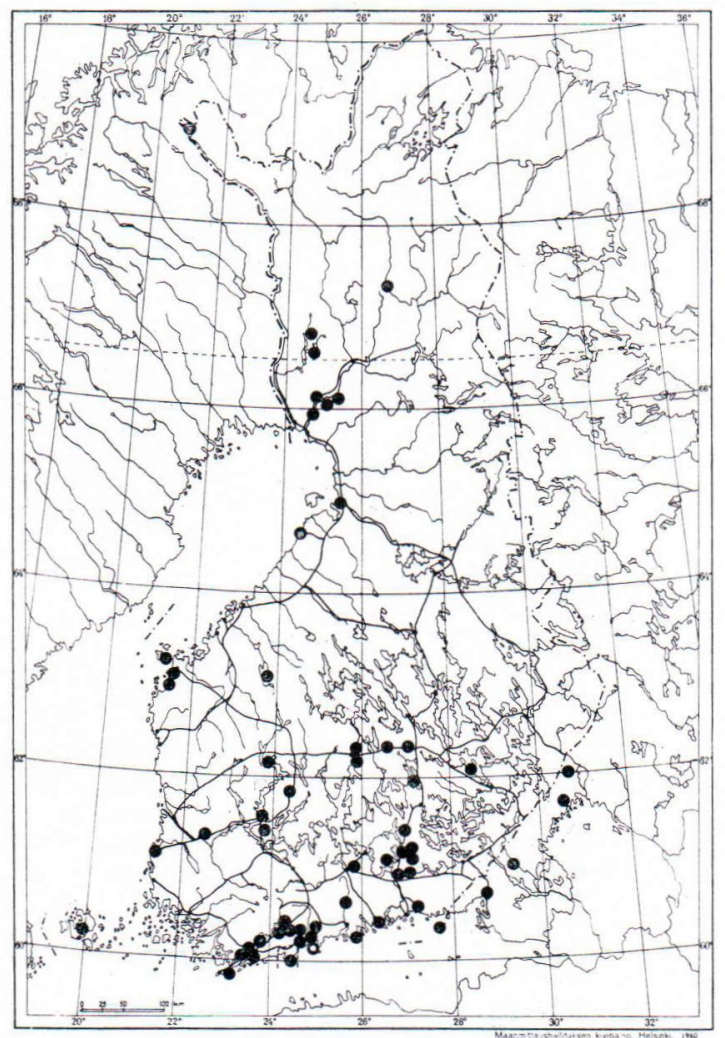

AвB. 7. Rezente Verbreitung von Cyclotella meneghiniana (Punkte) und C. $m$. var. laevissima (Ring). angetroffene, aber wahrscheinlich verhältnismässig häufige Art. Abb. 4. Individuumgröße in Finnland rel. klein. Subfossil in Yoldia- und Litorina-sowie Postlitorina-Ablagerungen.

\section{Stephanodiscus}

Diskus- bis trommelförmige Zellen, die entweder einzeln oder zu dichten Ketten vereinigt (S. hantzschii) leben. Valvarebene kreisförmig, verhältnismässig eben oder konzentrisch gewellt. Bei einigen Arten ist die Fläche von. Stacheln umrandet ( $S$. astraea, $S$. hantzschii). Kennzeichnend ist eine radiale Punkt- oder Rippenstruktur derart, da $\beta$ die in ihrem Mittelfeld lichtere Punktstruktur sich 2-4 fach oder in dicken Rippen fortsetzt.

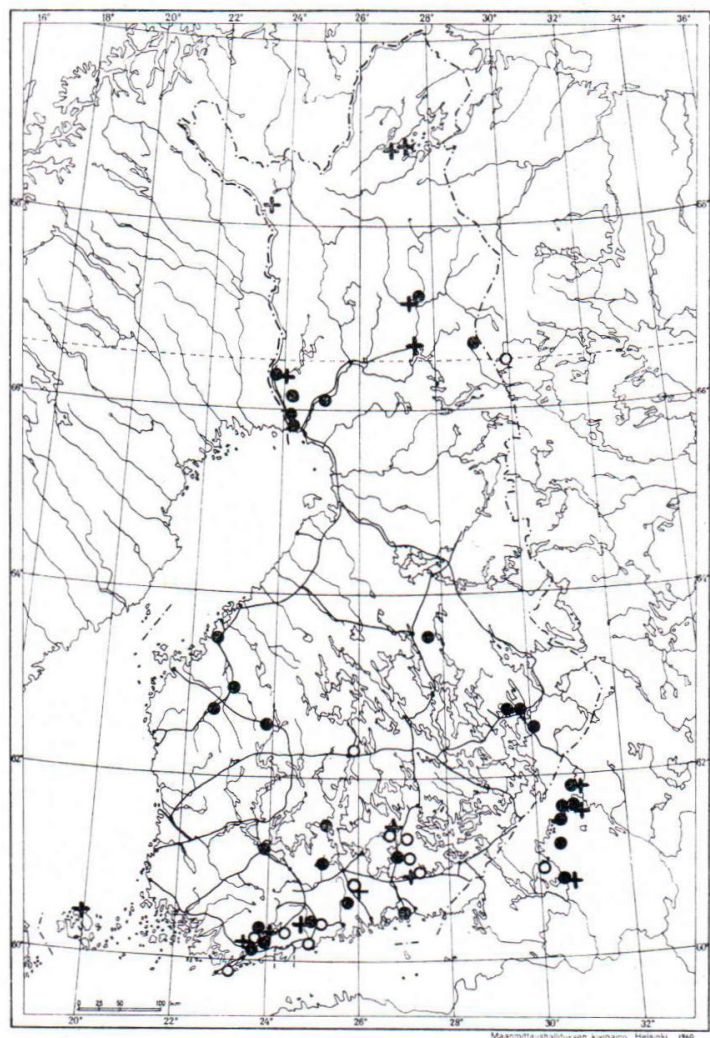

Авв. 8. Rezente Verbreitung von Stephanodiscus astraea (Punkte), S. a. var. minutula (Kreuze) und Stephanodiscus dubius (Ringe). 


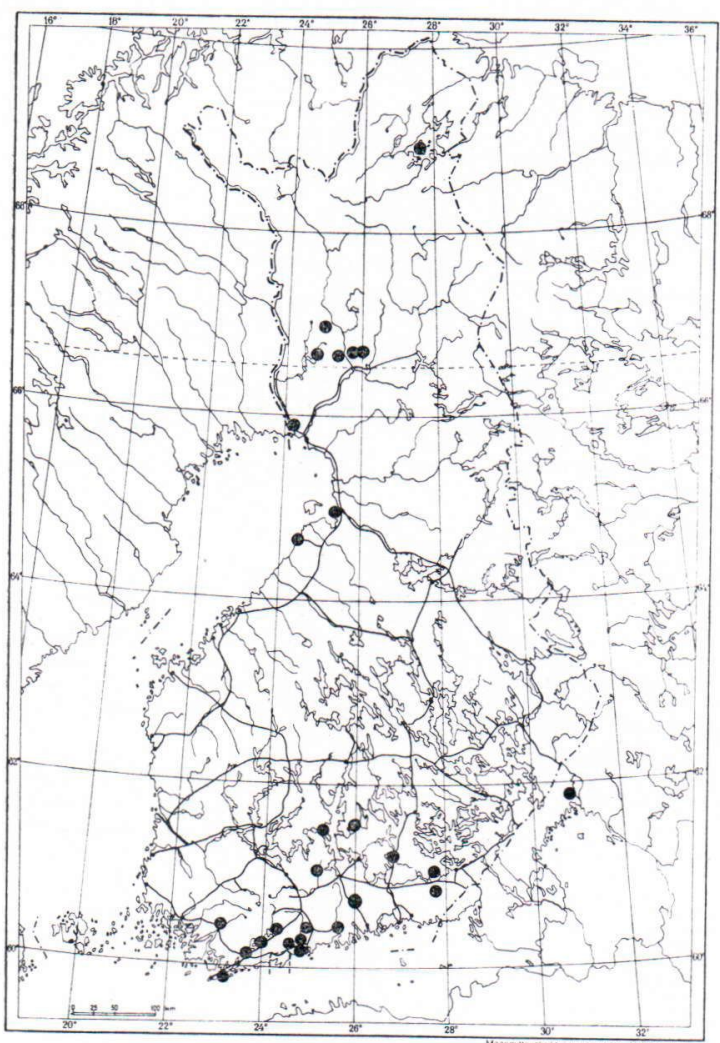

Авв. 9. Rezente Verbreitung von Stephanodiscus bantzschii (Punkte).

\section{Stephanodiscus alpinus Hustedt.}

Eine der $S$. astraea stark ähnelnde Form. Ein Merkmal, durch das sie sich von $S$. astraea unterscheidet, sind die in regelmässigen Abständen auftretenden Randdornen. Die Art ist eine Kaltwasserform (Huber-Pestalozzi 1942) und subfossil in Südfinnland in präborealer Ablagerung angetroffen worden (R. Aario 1965 b).

Stephanodiscus astraea (Ehr.) Grunow.

Synonyme: Discoplea astraea Ehr., Discoplea rotula Ehr., Cyclotella rotula Kütz., Cyclotella astraea Kütz., Stephanodiscus aegyptiacus Ehr., Stephanodiscus astraea var. spinulosa Grunow.

Häufige Planktonform von Grossseen. Die Art lebt auch in schwach salzhaltigem Küstenwasser (Cleve-Euler 1951, Mölder 1958). Abb. 8. Häufig in Süßwasser-, besonders Ancylussee-Sedimenten.
Var. minutula (Kütz.) Grun.

Synonyme: Cyclotella minutula Kütz., Discoplea oregonica Ehr., Cyclotella oregonica Ralfs, Stephanodiscus balatonica Pantocsek.

Erscheint oft neben der Hauptform im Plankton, ausserdem aber auch in kleineren Gewässern. Vielleicht eine Form kälterer Wässer als die Hauptform denn sie kommt in Lappland auch in denjenigen Seen vor, in denen die Hauptform nicht gefunden worden ist. Abb. 8. Subfossil häufig in Süßwasser-Sedimenten.

\section{Stephanodicus dubius (Fricke) Hustedt}

Synonyme: Cyclotella dubia Fricke, Stephanodiscus pulcherrimus A. Cleve, Cyclotella dubia var. spinulosa A. Cleve.

Häufig im Plankton eutropher Seen und auch in schwach brackigem Küstenwasser gedeihend. Abb. 8. Subfossil auch darum allgemein, weil die dicke Frustelstruktur das Auflösen besser vertragen hat.

Stephanodiscus bantzschii Grunow.

Synonyme: Cyclotella operculata Hantzsch, Stephanodiscus balticus Schumann, Stephanodiscus bantzschianus Grun., Stephanodiscus bantzsbii var. pusilla Grun., Stephanodiscus zachariasi Brun, Stephanodiscus hantzschii var. zachariasi Brun, Stephanodiscus bantzschii var. delicatulla A. Cleve, Stephanodiscus minor Reverdin.

Eine im Plankton eutropher und verunreiningter Seen gemeine Art, gedeiht aber auch in schwach salzhaltigem Wasser im Küstengebiet. Abb. 9. Infolge ihrer dünnen Zellwandung löst sie sich verhältnismässig empfindlich auf und erscheint in den Sedimenten nicht sehr häufig.

\section{Coscinodiscus}

Diskusförmige, im allgemeinen relativ große Zellen. Die Valvarfläche oft konzentrisch, selten tangential gewellt. Die in Finnland angetroffenen Arten zeigen an der Oberfläche eine punktierte oder areolierte Struktur, die auf bestimmte Weise, meistens radial angeordnet ist.

Coscinodiscus apiculatus Ehrenberg.

Ist nur von Tammisaari her aus Plankton vom Dezember 1936 als sehr selten dargestellt worden (Halme \& Mölder 1958). Abb. 10. Wahrscheinlich ein zufälliges Vorkommen, durch Meeres- 


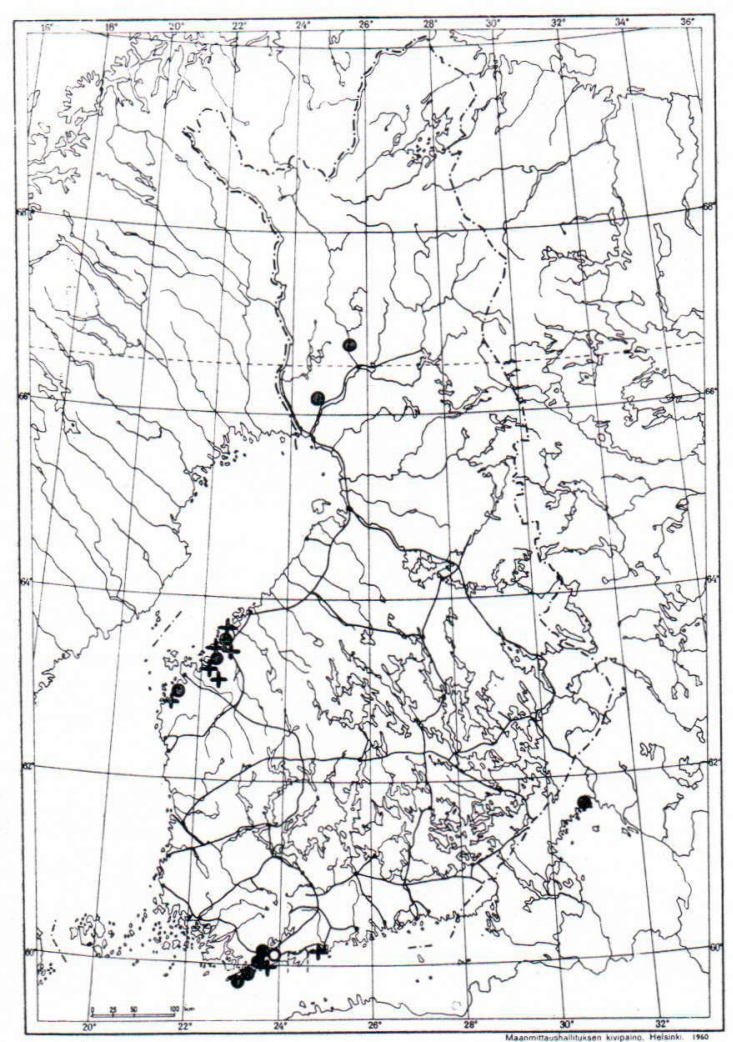

ABB. 10. Rezente Verbreitung von Coscinodiscus apiculatus (Ring), Coscinodiscus lacustris (Punkte) und $C$. $l$. var. septenrionalis (Kreuze).

strömungen oder Stürme in die Ostsee verfrachtet, denn die Art gehört offenbar nicht zu den eigentlichen Formen der Ostsee. Ebenso ist die Art als Seltenheit aus Yoldia- und Litorinaschichten angegeben.

\section{Coscinodiscus argus Ehrenberg.}

Synonyme: Coscinodiscus radiatus Ehr., Coscinodiscus crassus Bailey, Coscinodiscus irradiatus Harting, Coscinodiscus woodwardi Schmidt.

Marine Art, die als subfossil für eine aus der Endphase des Baltischen Eissees herrührende Ablagerung des Moores Mustalampi in Kämärä angeführt wird (Hyyppä 1937).

\section{Coscinodiscus asteromphalus Ehrenberg}

Synonyme: Coscinodiscus asteromphalus var. genuina Grunow, Coscinodiscus asteromphalus var. conspicua Grunow.

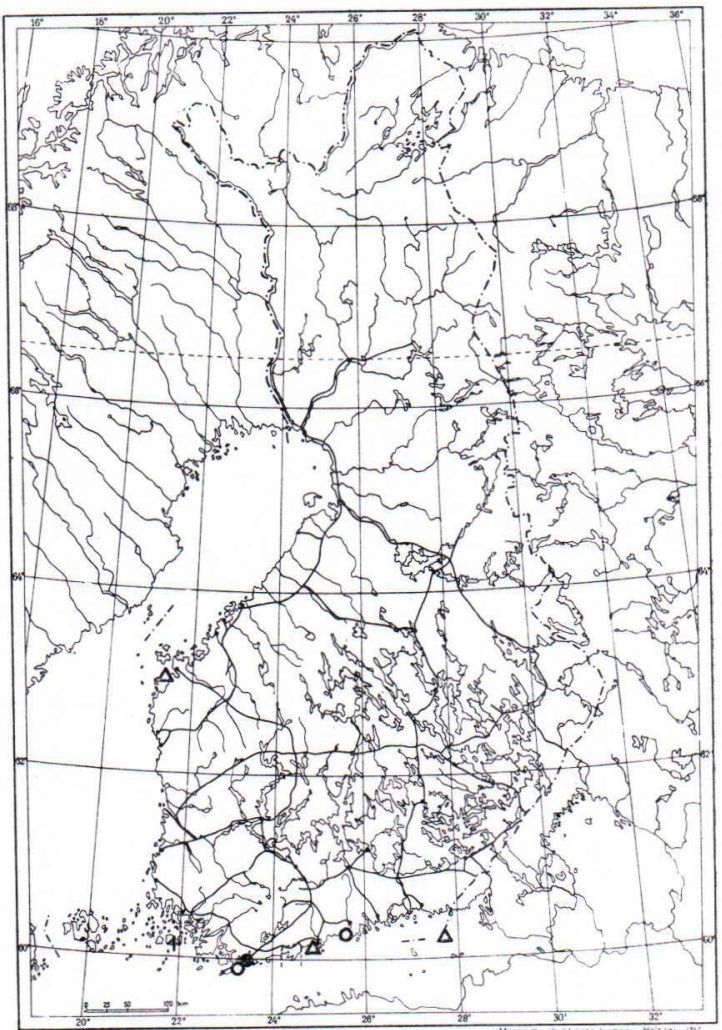

Авв 11. Rezente Verbreitung von Coscinodiscus asteromphalus (Kreutz), Coscinodiscus stellaris (Punkt), Actinocyclus ebrenbergii var. ralfsii (Ringe) und Chaetoceros muelleri (Drieecke).

Weltweite marine pelagische Planktonform (Hendey 1964), nach anderen Angaben ist die Art nicht pelagisch (u.a. Cleve-Euler 1951). Rezent im Südteil der Ostsee (Cleve-Euler op. cit.) und nördlich von Gotland (Mölder 1962a). Wahrscheinlich zeitweilig an die finnische Küste verschleppt. Die Art dargestellt aus zwei Schlammen eines geologisch kürzlich von der Ostsee abgeschnürten Weihers des westlich von Turku gelegenen Schärenhofes (Fontell 1926). Abb. 11. Als subfossil berichtet aus Kämärä, einer Ablagerung des Baltischen Eissees (Hyyppä 1937), aus einer Litorina-Ablagerung im Pasilansuo von Helsinki (Brander \& Brenner 1935), aus dem Tattarisuo (Hyyppä op. cit.) sowie von Åland (Cleve-Euler 1951), aus Ostbottnien (Backman \& 
Cleve-Euler 1922, Mölder 1964a) und NordSatakunta (Aario 1932). Verhältnismässig häufig in Litorina- und Postlitorina-Ablagerungen am Finnischen Meerbusen.

Coscinodiscus commutatus Grunow.

Synonyme: Coscinodiscus concinnus var. Jonesiana Rattray, Coscinodiscus radiatus var. jonesiana Van Heurck, Coscinodiscus biconicus Van Breemen, Coscinodiscus jonesianus var. commutata (Grun.) Hustedt.

Marine Art, die auch in Brackwasser gedeiht. Rezent selten und vielleicht zufällig im Küstenplankton des Finnischen Meerbusens. Subfossil in Litorina- und Postlitorina-Ablagerungen selten.

Coscinodiscus curvatulus Grun. var. karianus $\mathrm{Cl} \&$ Grun.

Marine Form, die im Weiher Rimpilampi (Backman \& Cleve-Euler 1922) und in Sievi (Cleve-Euler 1951) angetroffen worden ist. Wahrscheinlich sind die betr. Vorkommen interglazialer Herkunft.

Coscinodiscus divisus Grunow.

In Finnland nur bei Tammisaari (Mölder 1943a, Halme \& Mölder 1958) und vor Helsinki (Mölder 1944a) festgestellt. Abb. 12. Nach Hustedt (1931) ist die Art marin und wahrscheinlich neritisch oder litoral.

Coscinodiscus excentricus Ehrenberg.

Synonyme: Odontodiscus excentricus Ehr., Coscinodiscus labyrintbus Roper, Coscinodiscus minor A. Schmidt, Thalassiosira excentrica Cleve, Coscinodiscus beliozoides Siddall.

Vielgestaltige Art; von ihren Formen ähnelt eine besonders $C$. lineatus var. irregularis. Möglicherweise liesse sich letztere der Hauptart als Synonym anschließen.

Marine Art, die nicht zu den eigentlichen Formen der Ostsee gehört, sondern in salzige Meere eingeht. Subfossil aus Laihia, einer LitorinaAblagerung (Mölder 1946a), und einem Interglazialsediment von Rouhiala. (Brander 1941). Var. fasciculata Hustedt.

Synonym: Coscinodiscus subtilis Ehrenberg

Ein für die Ostsee sehr seltenes Vorkommen, das u.a. vor Hanko und nördlich von Gotland in Grundschlamm (Halme \& Mölder 1958, Mölder 1962a) sowie westlich von Helsinki in Winter-

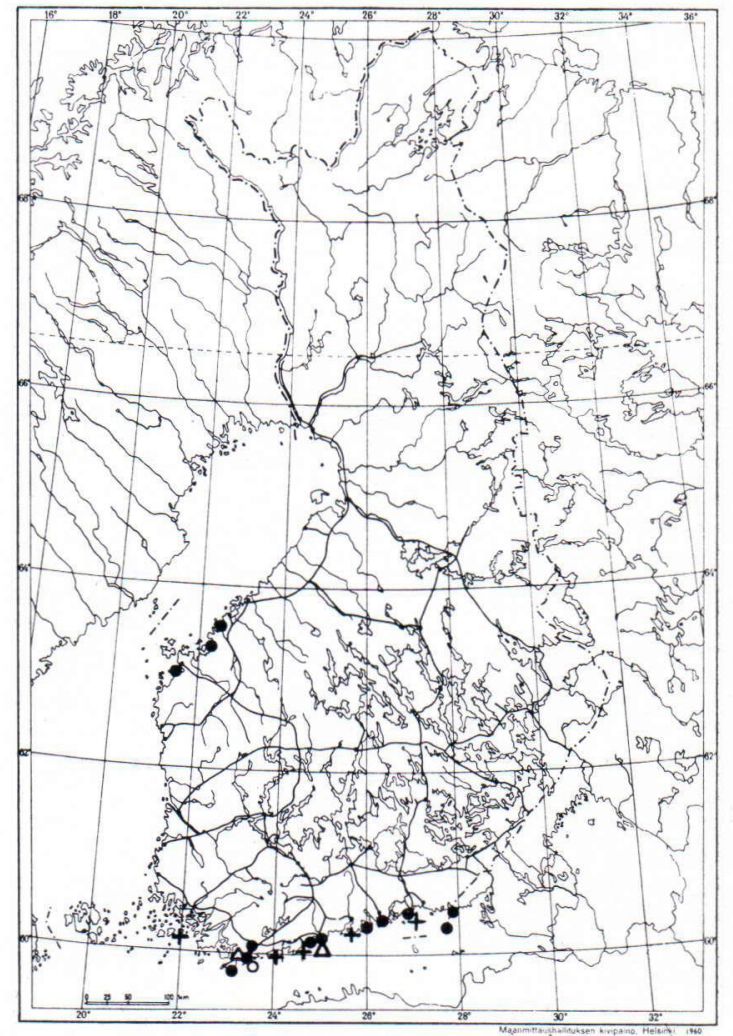

Авв 12. Rezente Verbreitung von Coscinodiscus divisus (Dreiecke), Coscinodiscus lineatus (Ring), Actinocyclus ebrenbergi (Punkte) und $A$. e. var. crassa (Kreuze).

plankton der Strandzone (Mölder \& Tynni 1966) angetroffen worden ist.

Coscinodiscus granii var. araliensis (Ostf.) Hustedt.

Im Aral-See pelagische Form, die auf für die Nordsee belegt ist (Hustedt 1930), die Hauptform für die SW-Ostsee (Cleve-Euler 1951). Die Variation ähnelt der kleinareolierten Form Coscinodiscus asteromphalus. Sie, selten auch die Hauptform, ist u.a. aus einer Litorina-Ablagerung von Pyhtää dargestellt (Hyyppä 1937).

Coscinodiscus kütringi A. Schmidt.

Synonym: Coscinodiscus marginatus A. Schmidt

Eine Diatomee nord- und westeropäischen Küstengebiets (Hustedt 1930). Im Oberflächenteil des Grundes der südlichen Ostsee (Mölder 1964a) sowie subfossil u.a. in einer LitorinaAblagerung vor Pori angetroffen. 
Coscinodiscus lacustris Grunow.

Synonyme: Cyclotella punctata W. Smith, Stephanodiscus punctatus Grunow.

Pelagische Art schwach brackigen Wassers, aber auch in Süßwasser gedeihend. Die Beobachtungen konzentrieren sich jedoch auf das Küstengebiet (Abb. 10). Subfossil besonders in LitorinaSedimenten.

Coscinodiscus lacustris var. septentrionalis Grunow.

(Synonym: Coscinodiscus lacustris var. byperborea Grunow in Hustedt 1930.)

Art mit grösseren Areolen, lebt pelagisch in Brackwasser, u.a. im Finnischen und im Bottnischen Meerbusen. Abb. 10. Häufig in LitorinaAblagerungen, häufiger als die Hauptform.

\section{Coscinodiscus lineatus Ehrenberg.}

Synonyme: Coscinodiscus ebrenbergi O'Meara, Coscinodiscus macraeanus Grun., Coscinodiscus leptopus Grunow.

Nur einmal in der Nähe von Hanko angetroffen (Halme \& Mölder 1958). Abb. 12. Gehört nicht zu den eigentlichen rezenten Formen der Ostsee, sondern hauptsächlich zu den südlichsten Meeren (Hendey 1937). Subfossil in einer Interglazialablagerugn von Rouhiala angetroffen.

Var. irregularis Temp. et Perag.

Synonym: Coscinodiscus leptopus var. irregularis Temp. et Perag.

Einer Form von Coscinodiscus excentricus ähnlich, aber größere Übereinstimmungen verbinden mit C. lineatus. Die Variation ist stellenweise verhältnismässig gemein in Litorina-Ablagerung vor Pori gefunden worden.

Coscinodiscus marginatus Ehrenberg.

Synonyme: Coscinodiscus limbatus Ehr., Coscinodiscus fimbriatus-limbatus Ehr., Coscinodiscus robustus A. Schmidt, Coscinodiscus radiatus f. beterosticta Grun., Coscinodiscus radiatus var. subaequalis Grun. f. parva Pantocsek.

Weitverbreitet marine Form, die in der Ostsee sehr selten angetroffen worden ist, wie im Oberflächenteil des Ostseegrundes (Mölder 1962), nahe bei Hanko in Pojo-Bucht (Mölder 1943a) und Kemi. Abb. 13. Subfossil ebenfalls selten in Yoldia- und Litorina-Sedimenten.

Coscinodiscus niditus Greg.

Marine Benthosform, subfossil belegt aus dem Moor Takkasuo von Kajaani (Kilpi 1937). Wahrscheinlich interglazialer Herkunf.

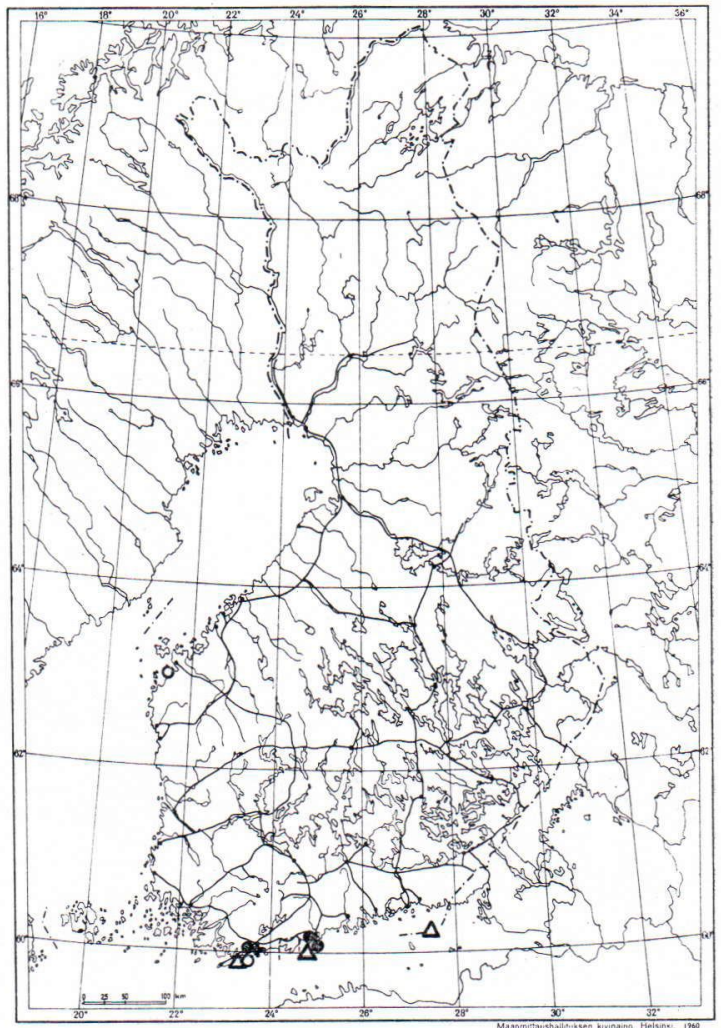

Авв. 13. Rezente Verbreitung von Coscinodiscus marginatus (Kreuz) Coscinodiscus oculus iridis (Punkte), Coscinodiscus perforatus (Dreicke) und Coscinodiscus radiatus (Ringe).

Coscinodiscus nodulifer A. Schmidt.

In Litorina-Ablagerung bei Helsinki (Mölder 1944a) und vor Pori aufgefunden.

Coscinodiscus oculus iridis Ehrenberg.

Weitverbreitete marine Form, die selten auch an der Küste des Finnischen Meerbusens beobachtet worden ist. Abb. 13. Subfossil besonders in Litorinasedimenten.

Coscinodiscus perforatus Ehrenberg.

Synonym: Coscinodiscus woodwardi Eulenstein.

Im Nordseeplankton häufige Art (Hendey 1964), aber die Beobachtungen über die Ostsee beschränken sich auf seltene Vorkommen an der Küste des Finnischen Meerbusens. Abb. 13. Subfossil in Sedimenten des Yoldia- und des Litorinameeres.

Var. cellulosa Grunow. 
Subfossil aus Kajaani belegt (Kilpi 1937). (Die als Coscinodiscus oculus iridis abgebildete Form aus der Bucht Otsolahti westlich von Helsinki (Mölder \& Tynni 1966, Tafel 1, Fig. 10b) ist wahrscheinlich Cocs. perforatus var. cellulosa.) Coscinodiscus plicatus Grun.

Dem Coscinodiscus lacustris ähnliche Form, unterscheidet sich aber von ihm vorwiegend durch die geraden radialen Areolenreihen. Belegt aus Litorina-Ablagerung von Tervola in Ostbottnien (Mölder 1946).

Var. byperboreus (Grun.) A. Cl.

Synonym: C. lacustris var. byperboreus Grun.

Cleve-Euler (1951) hat die Variation in N-Satakunta in Litorina-Ablagerung von Panelia erkannt.

Coscinodiscus radiatus Ehrenberg.

Synonyme: Coscinodiscus borealis Ehr., Coscinodiscus fallax Schumann, Coscinodiscus caspius Ehr., Coscinodiscus radiatus f. minor A. Schmidt, Coscinodiscus radiolatus A. Schmidt, Coscinodiscus devius A. Schmidt, Coscinodiscus radiatus $\mathrm{f}$. parva Grun., Coscinodiscus radiatus var. subaequalis Grun., Coscinodiscus radiatus var. borealis Grun., Coscinodiscus radiatus var. glacialis Grun., Coscinodiscus radiatus var. media Grunow.

Marine Art, die in der Ostsee zufällig vorkommt. Aus der Nähe von Hanko (Mölder 1943, Halme \& Mölder 1958) und Vaasa (Abb.

13) sowie als selten aus Proben vom Oberflächenteil des Ostseegrundes (Mölder 1962) dargestellt. Subfossil in Litorina-Sedimenten selten.

Coscinodiscus rotbii var. normanni V. Heurck.

Synonyme: Coscinodiscus normani Gregory, Coscinodiscus fasciculatus O'Meara, Coscinodiscus subtilis var. normani Van Heurck.

Brackwasserform, die subfossil u.a. aus dem Takkasuo von Kajaani belegt ist (Kilpi 1937). Coscinodiscus stellaris Roper.

Synonyme: Coscinodiscus stellaris var. fasciculata Castracane.

Marine Form, die nur einmal bei Hanko beobahtet worden ist (Halme \& Mölder 1958). Abb. 11. Var. symbolophora ebenfalls in der Nähe von Hanko festgestellt (op. cit.).

\section{Actinoptychus}

Diskusförmige Zellen, deren dekorative Ober- flächenstruktur aus sektorartig angeordneten, abwechselnd in verschiedenen Ebenen gelegenen Strukturfiguren besteht, die aus Punktierung und Areolenfiguren zusammengesetzt sind. Mittelfeld hyalin.

Actinoptychus undulatus (Bailey) Ralfs.

Eine in alle nördlichen gemässigten Meere verbreitete Form. Besonders an der Küste der Britischen Inseln häufig im neritischen Plankton (Hendey 1964). Selten vertreten im Oberflächenteil des Bodens der südlichen Ostsee, subfossil im Moore Takkasuo von Kajaani (Kilpi 1937), in Paltoma am Oulujoki, im See Höytiäinen (Cleve-Euler 1951), in Rouhiala (Brander 1941) und in Somero (Gardemeister 1968). Die subfossilen Funde sind wahrscheinlich interglazialer Herkunft.

\section{Auliscus}

Diskusförmige Zellen mit rundlich elliptischer Valvarfläche. Deren Struktur ist im allgemeinen zygomorph angeordnet, bestehend aus zwei runden abgegrenzten Erhebungen und einer von ihnen rechtwinklig bis schräg ausgehenden Liniierung und anderseits einer am hyalinen Mittelfeld ainsetzenden Striemelung.

Auliscus caelatus Bailey.

Im ganzen europäischen Meeresgebiet verbreitet (Hustedt 1930), aber nicht in der Ostsee. In den Interglazialablagerungen von Rouhiala (Brenner 1941) und Somero (Gardemeister 1968) angetroffen.

Auliscus sculptus W. Sm. Ralfs.

Marine Litoralform, die aus Finnland subfossil, aus Litorina-Ablagerung des Moores Sikovuori nördlich von Hamina dargestellt worden ist (Hyyppä 1937: Auliscus sp., Cleve-Euler 1951: Auliscus sculptus).

\section{Actinocyclus}

Diskusförmige Zellen, deren Valvarfläche radial areoliert ist bis zur Randzone, wo die Struk- 
tur feiner, maschig wird. In der Randzone der Strukturtypen ein Auge, an der Peripherie Randdornen.

\section{Actinocyclus ebrenbergi Ralfs.}

Synonyme: Actinocyclus nebulosus Peragallo. Nach der Zahl der radialen Bündel hat Ehrenberg zahlreiche Formen unterschieden, u.a. Actinocyclus octonarius.

An der Ostsee und in Küstengebieten häufige Diatomee von Brack- und Salzwasser. Subfossil in Yoldia- und Litorina-Meerablagerungen, in letzteren häufig.

Var. crassa (W. Smith) Hustedt.

Synonyme: Eupodiscus crassus W. Sm., Actinocyclus crassus van Heurck, Actinocyclus subcrassus Rattray.

Eine kleinere Form als die Hauptform, sie erscheint mit dieser zusammen an denselben Stellen, aber selten.

Var. ralfsii (W. Smith) Hustedt.

Synonyme: Eupodiscus ralfsii W. Sm., Coscinodiscus fuscus Norman, Actinocyclus fuscus A. L. Smith, Actinocyclus ralfsii Ralfs.

Rezent vor Hanko beobachtet (Mölder 1943a). Subfossil oft mit der Hauptform zusammen.

\section{Rhizosolenia}

Zylinderförmige oder platte längliche dünnwandige Zellen, deren Valvarflächen sich zu asymmetrisch und platt trichterförmigen sowie oft an den Enden stachelförmigen Gebilden gedehnt haben. Schuppen- oder halbringförmige Zwischenbänder bilden charakteristische Figuration.

Rbizosolenia calcar avis M. Schultze.

Marine Art, deren stachelförmige Teile sich offenbar in Sedimenten erhalten. In LitorinaSedimenten im Küstengebiet Finnlands häufig. Artbestimmung insofern unsicher, als die Diatomee sich in Sedimenten nicht unversehrt erhält. Rbizosolenia eriensis H. L. Smith

Im Plankton von Seen, Weihern und Flüssen angetroffene Art. Subfossil infolge der Schwäche der Zellwandung nicht festgestellt.

\section{Rhizosolenia longiseta Zacharias}

Die Art in vielen Seen und Flüssen verschiedenen Trophiegrades im Sommer in Plankton süd- lich des Polarkreises festgestellt (u.a. Levander 1904). Die häufigste der angetroffenen Rhizosolenia-Arten.

Rhizosolenia minima Levander

Synonym: Monoceros isthmiiforme van Goor

Art schwach brackigen Wassers, die vor Helsinki (Välikangas 1926) und Viipuri (Levander 1904) sowie in der Pojo-Bucht (Levander 1915) gefunden worden ist.

\section{Chaetoceros}

Dünnwandige kurze Zellen, deren Valvarfläche elliptisch oder rundlich elliptisch ist. Ihre apikalen Enden tragen meistens dickere oder dünnere Borsten, mittels deren sich die Zellen gewöhnlich miteinander verbinden und Kolonien bilden. Die Zellhälften sind oft verschieden. Infolge ihrer zarten Strucktur erhalten sich die Chaetoceros-Arten im allegemeinen nicht in den Sedimenten, ausser den Dauersporen, von denen viele identifizierbar sind. Alle Arten sind marin. Chaetoceros danicus Cleve

Meist einzeln, selten in kurzen Ketten wachsende Zellen (Hustedt 1930). Salz- und Brackwasserform, die im Küstenplankton Finnlands selten angetroffen worden ist, u.a. in der PojoBucht (Levander 1915, Mölder 1943) und in Helsinki (Välikangas 1926). Abb. 14.

Chaetoceros debilis Cleve

Planktonform salzigen Wassers, die aus dem Bottnischen Meerbusen (Leegaard 1920) und der Bucht Otsolahti (Mölder \& Tynni 1966) dargestellt worden ist.

\section{Chaetoceros densus Cleve}

Aus der Bucht Otsolahti und vor der Insel Lehtisaari berichtete Art (Mölder \& Tynni 1966). Die Art ähnelt $C$. danicus. Die betr. Arten sind schwer voneinander $\mathrm{zu}$ unterscheiden, und eine sicherer Bestimmung setzte weitere Beobachtungen voraus.

\section{Chaetoceros bolsaticus Schütt}

Kaltwasserform, die u.a. an der Küste des Finnischen und Bottnischen Meerbusens als Kolonien in Brack- und Salzwasser lebt (Välikangas 
1926, Purasjoki 1947, Halme \& Mölder 1958). Abb. 14.

Chaetocoros mitra (Bail.) Cleve

Eine besonders an der mitteleuropäischen Küste häufige Art (Hustedt 1930). Dauersporen kommen verhältmässig allgemein u.a. in Litorina-Ablagerungen vor Pori und am Finnischen Meerbusen vor. Vorhanden u.a. im Interglazialsediment von Rouhiala.

Chaetoceros muelleri Lemmermann

Eine im Brackwasser in Plankton als einzelne Zellen vorkommende und aus Finnland als selten dargestellte Art. Abb. 11.

Chaetoceros subsecundus (Grun.) Hustedt

An der Küste Europas neritisch lebende eurytherme Art (Hustedt 1930). Als Dauersporen in Litorina-Ablagerungen u.a. am Finnischen Meerbusen und vor Pori angetroffene Art.

\section{Chaetoceros subtilis Cleve}

Planktonform von Brack- und Salzwasser, die in Bottnischen Meerbusen, vor Hanko (Purasjoki 1947) und vor Helsinki (Välikangas 1926) zeitweilig zahlreich aufgefunden worden ist. Abb. 14. Chaetoceros wighami Brightw.

Synonym: Chaetoceros bottnicus Cleve

Pelagische Brackwasserform, die nach den Beobachtungen Levanders und Välikangas' an den finnischen Küsten besonders im Sommarplankton häufig vorkommt und oft im Herbst ein schwächeres Maximum bildet (Välikangas 1926, Purasjoki 1947). Juli 1963 bildete die Art u.a. die Mehrheit westlich von Porvoo vor dem Flusse Mustijoki. Auch im Plankton des Bottnischen Meerbusens häufig angetroffen (Vuorentaus 1913) Abb. 14.

\section{Attheya}

Platte zylinderförmige zarte Zellen mit ringförmigen Zwichenbändern und zwei endständigen Borsten.

\section{Attheya zachariasi J. Brun}

Synonym: Acanthoceras magdeburgense Honingmann.

Planktonform süssen Wassers, die besonders im Sommer in gleichartigen Gewässern wie

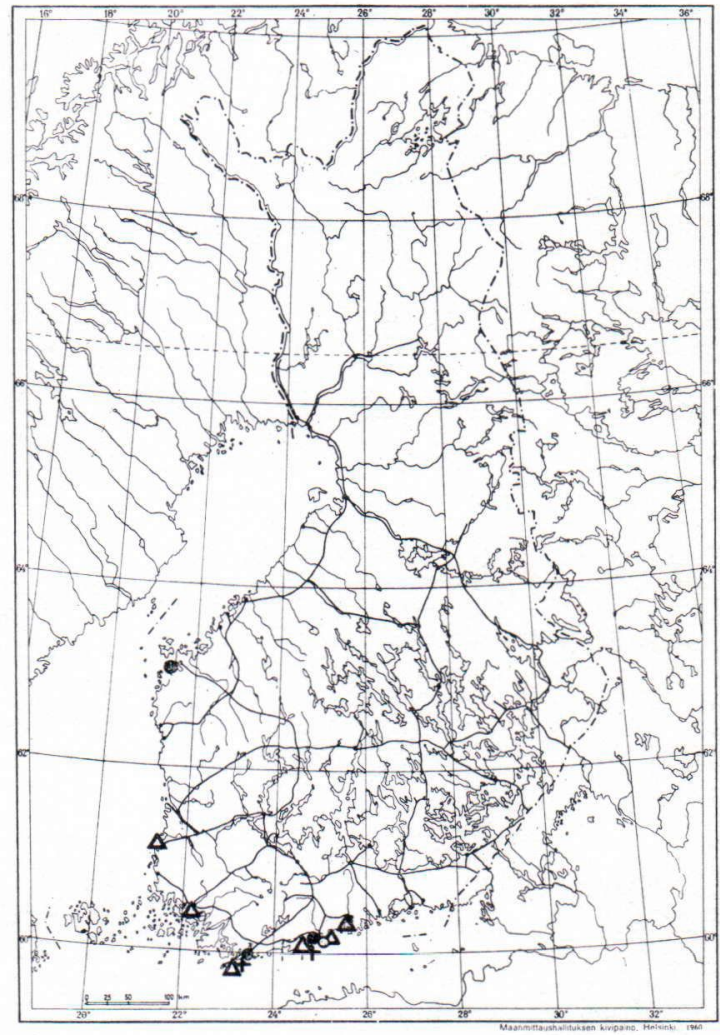

AвB. 14. Rezente Verbreitung von Chaetoceros danicus (Punkte), Chaetoceros bolsaticus (Kreuze), Chaetoceros subtilis (Ring) und Chaetoceros wighami (Dreiecke).

Rhizosolenia longiseta auftritt, wenn auch in bedeutend geringeren Mengen als diese. Auch in Brackwasser des Finnischen Meerbusens bei Viipuri angetroffen (Levander 1904). Hält sich subfossil nicht.

\section{Terpsinoe}

Die Kieselschalen bestehen aus länglichen, wellenförmig ovalen Valvarflächen, an deren eingeschnürten Stellen Septen liegen, sowie aus verhältnismässig hohen Gürtelbändern.

Terpsinoe americana (Bail.) Ralfs

Litorale Brackwasserform, die bei Porvoo im Bodenschlamm des kürzlich vom Finnischen Meerbusen abgeschnürten Sondby-Sees aufgefunden worden ist (Fontell 1926). Subfossil sehr selten festgestellt. 
TAFEL I
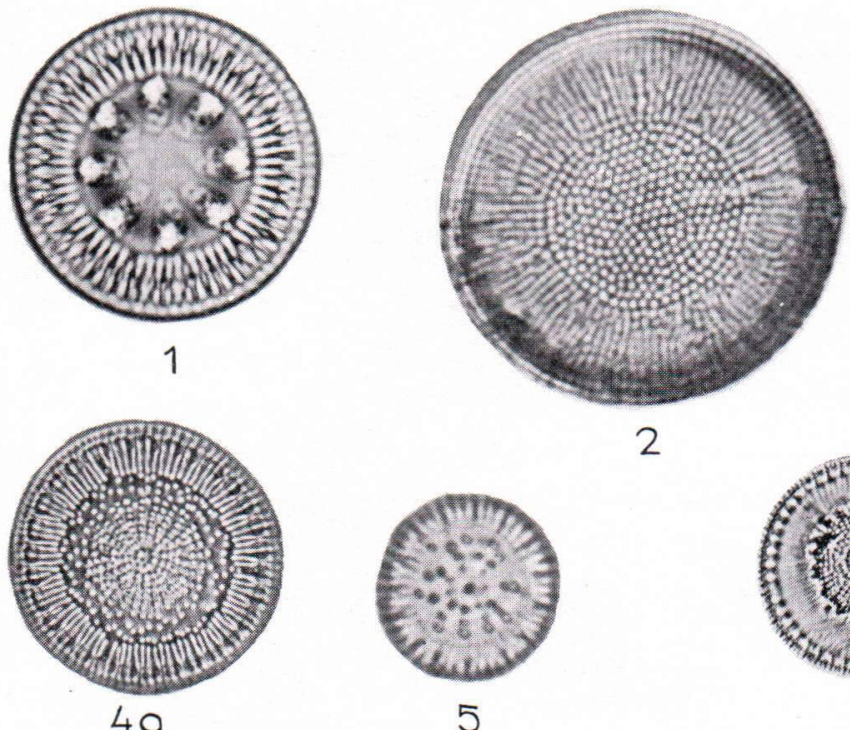

2
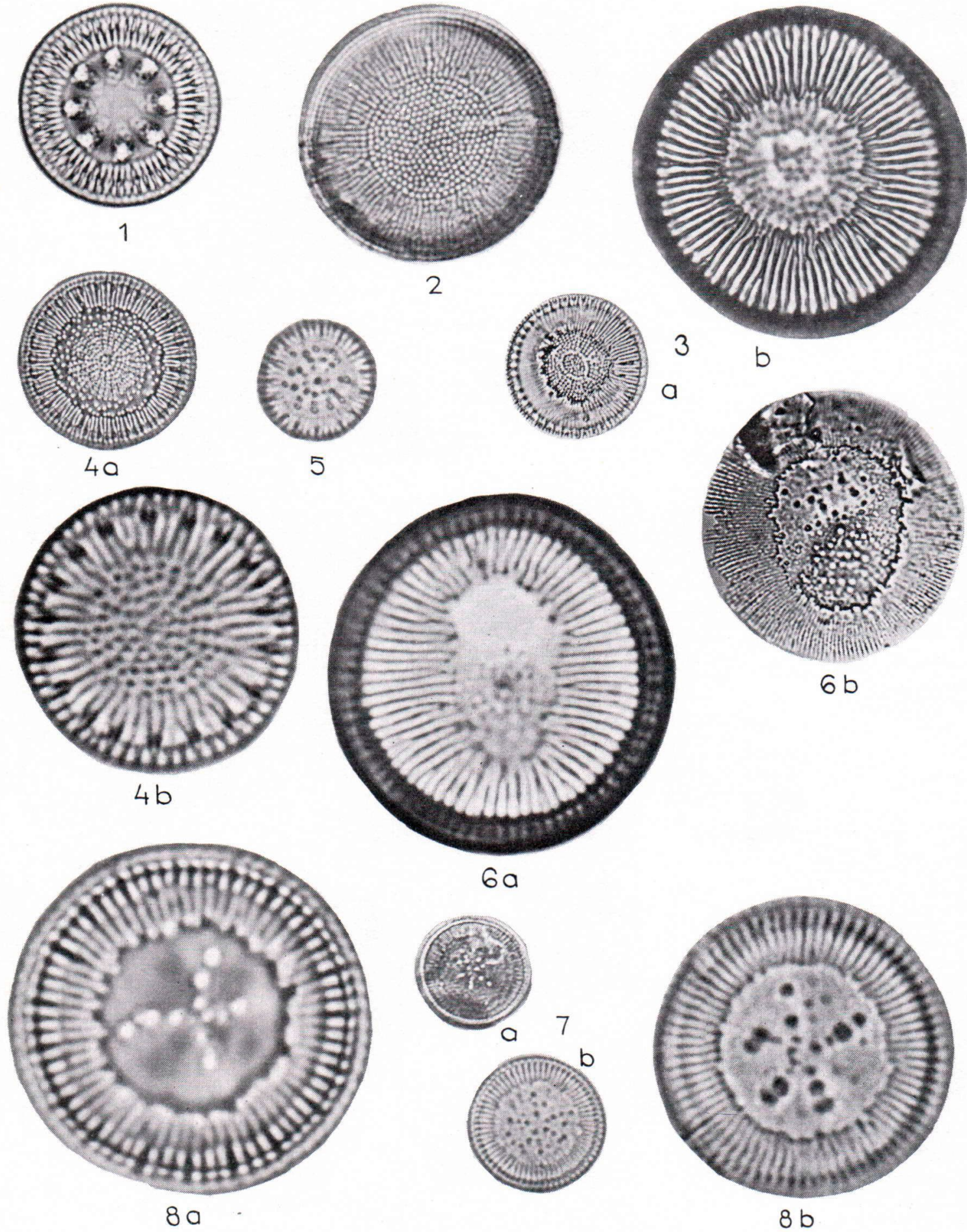

$6 a$

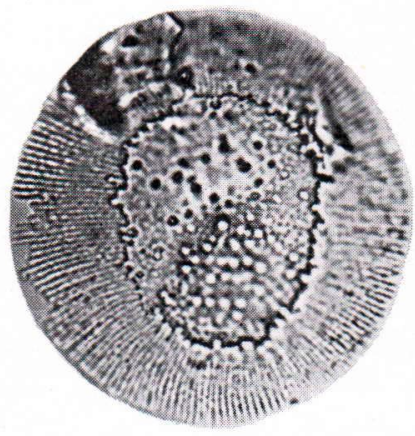

$6 b$

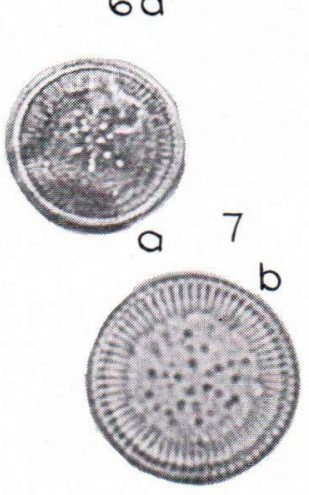

Fig. 1. Cyclotella antiqua, 2. C. arentii, 3. a-b. C. bodanica, 4. a-b. C. comta, 5. C. c. var. oligactis, 6. a-b. C. iris, 7. a-b. $C$. kutzingiana, Übergangsform nach var. radiosa, 8 , a-b. $C$. k. var. radiosa. 
T A FE L II
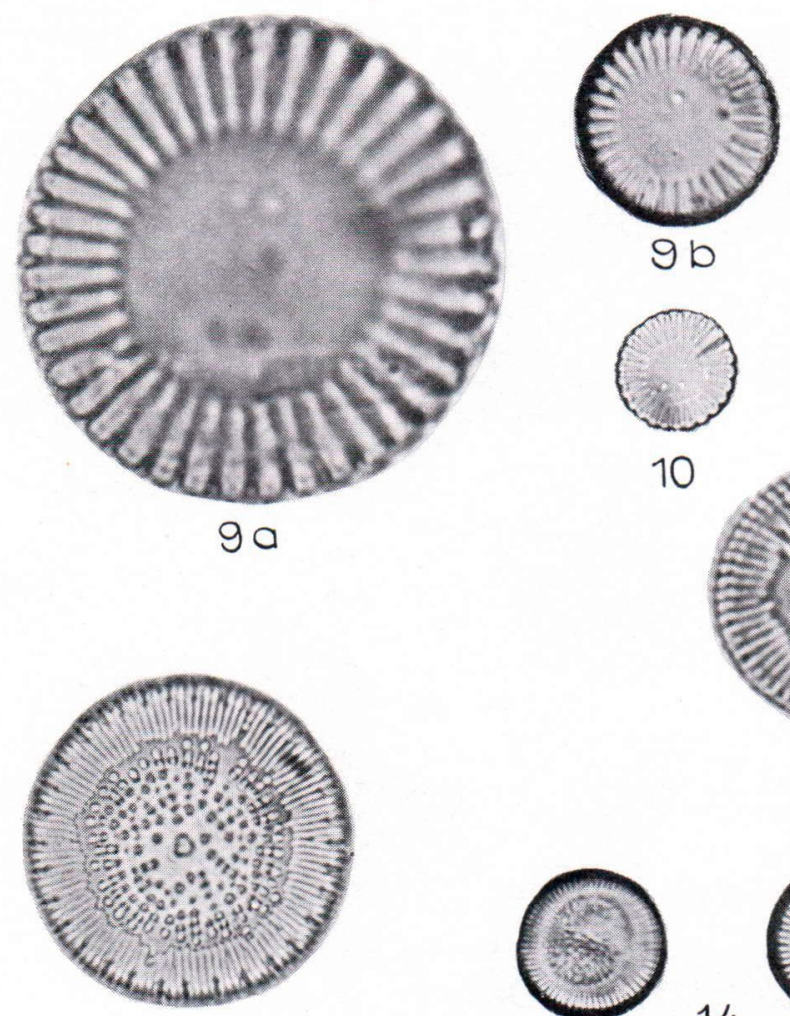

12
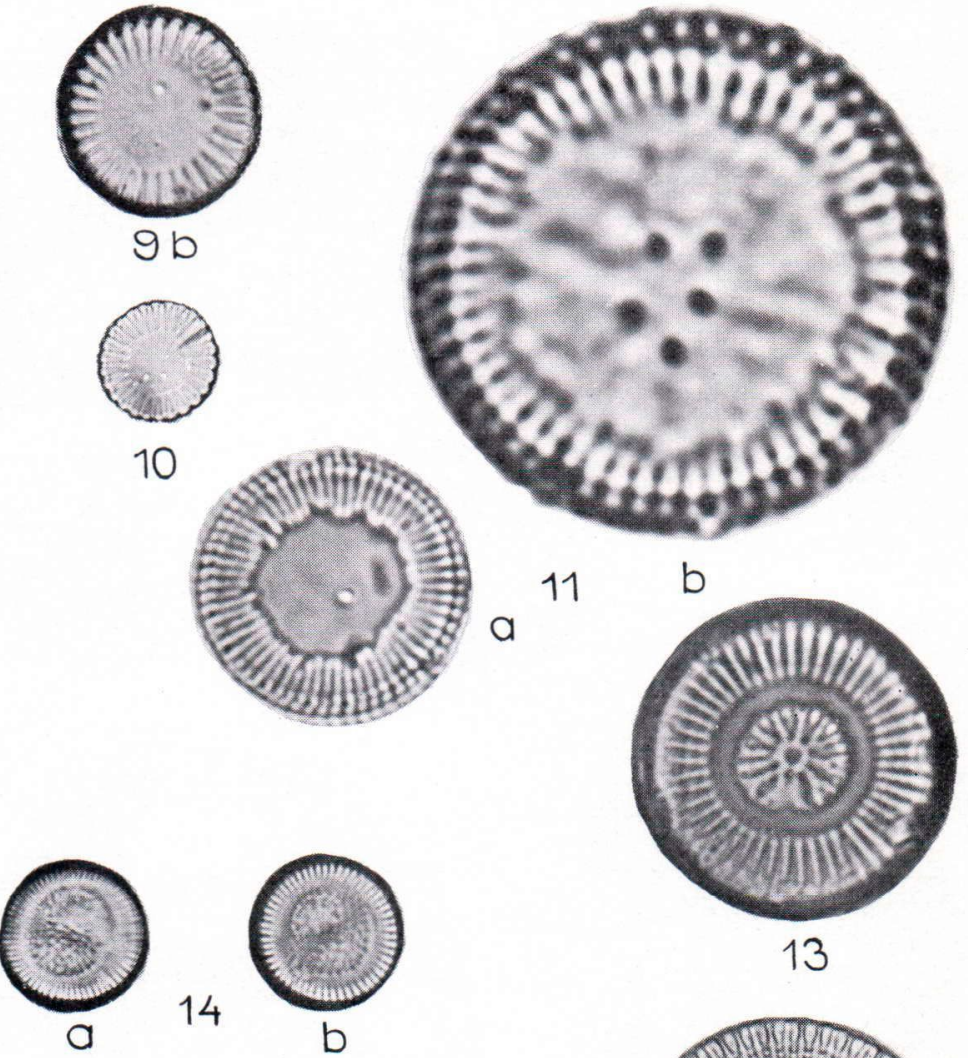

13

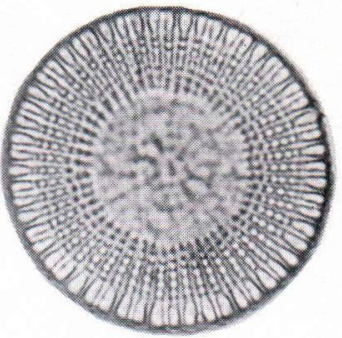

18
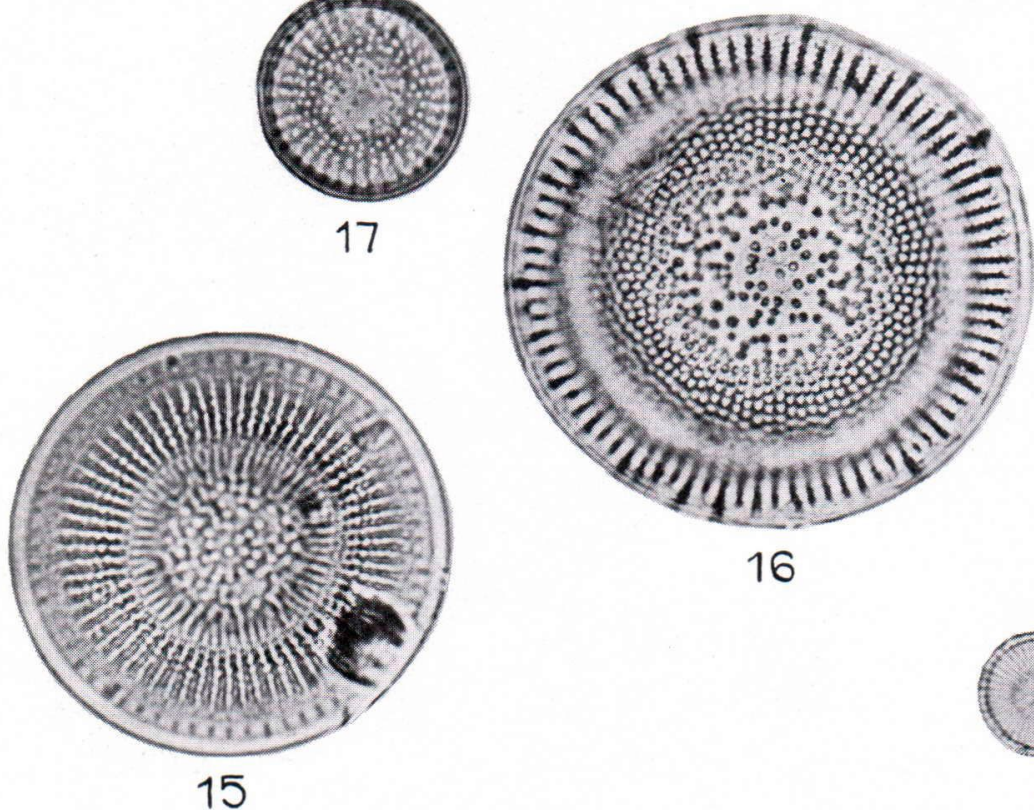

Fig. 9. a-b. Cyclotella meneghiniana, 10. C. m. var. laevissima, 11. a-b. C. ocellata, 12. C. quadriiuncta, 13. C. stelligera, 14. a-b. C. striata, 15. Stephanodiscus alpinus, 16. S. astraea, 17. S. a. var. minutula, 18. S. dubius, 19. a-b. S. bantzschii. 
TAFEL III
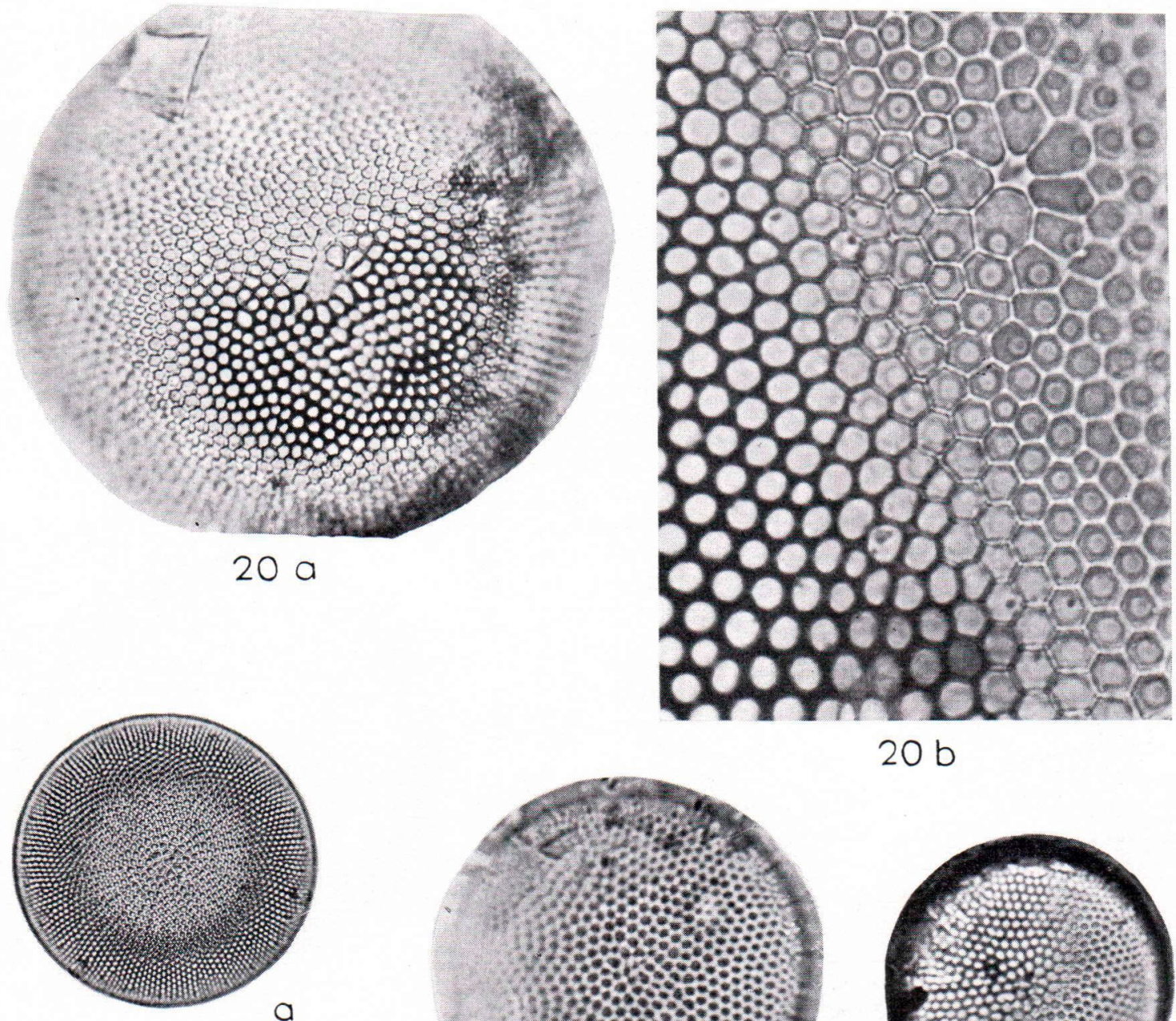

$$
20 \mathrm{~b}
$$

21
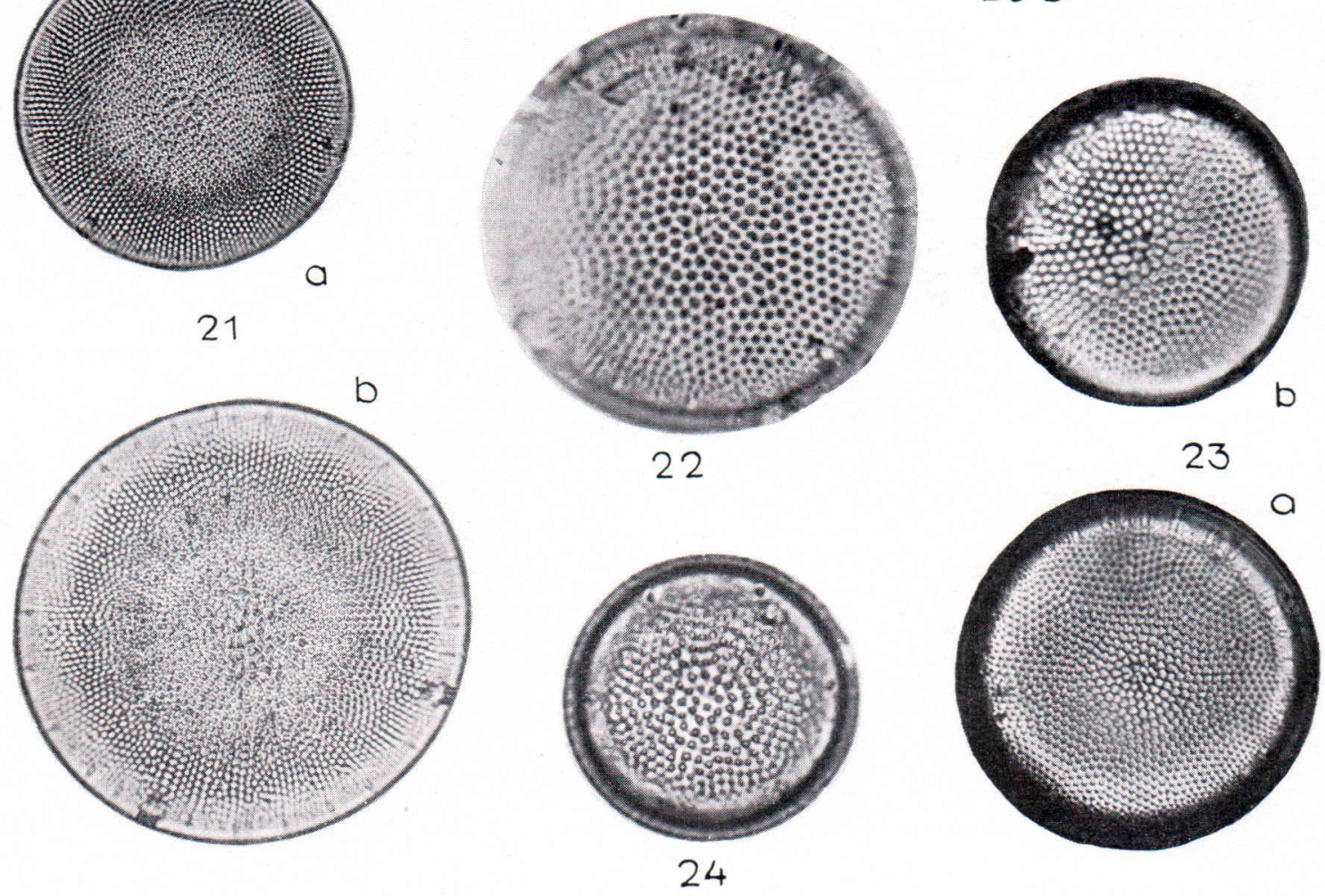

Fig. 20. a-b. Coscinodiscus asteromphalus, 21. a-b. C. commutatus, 22. C. excentricus, 23. a-b. C. e. var. fasciculata, 24. C. kiitzingi. 
TA FEL IV
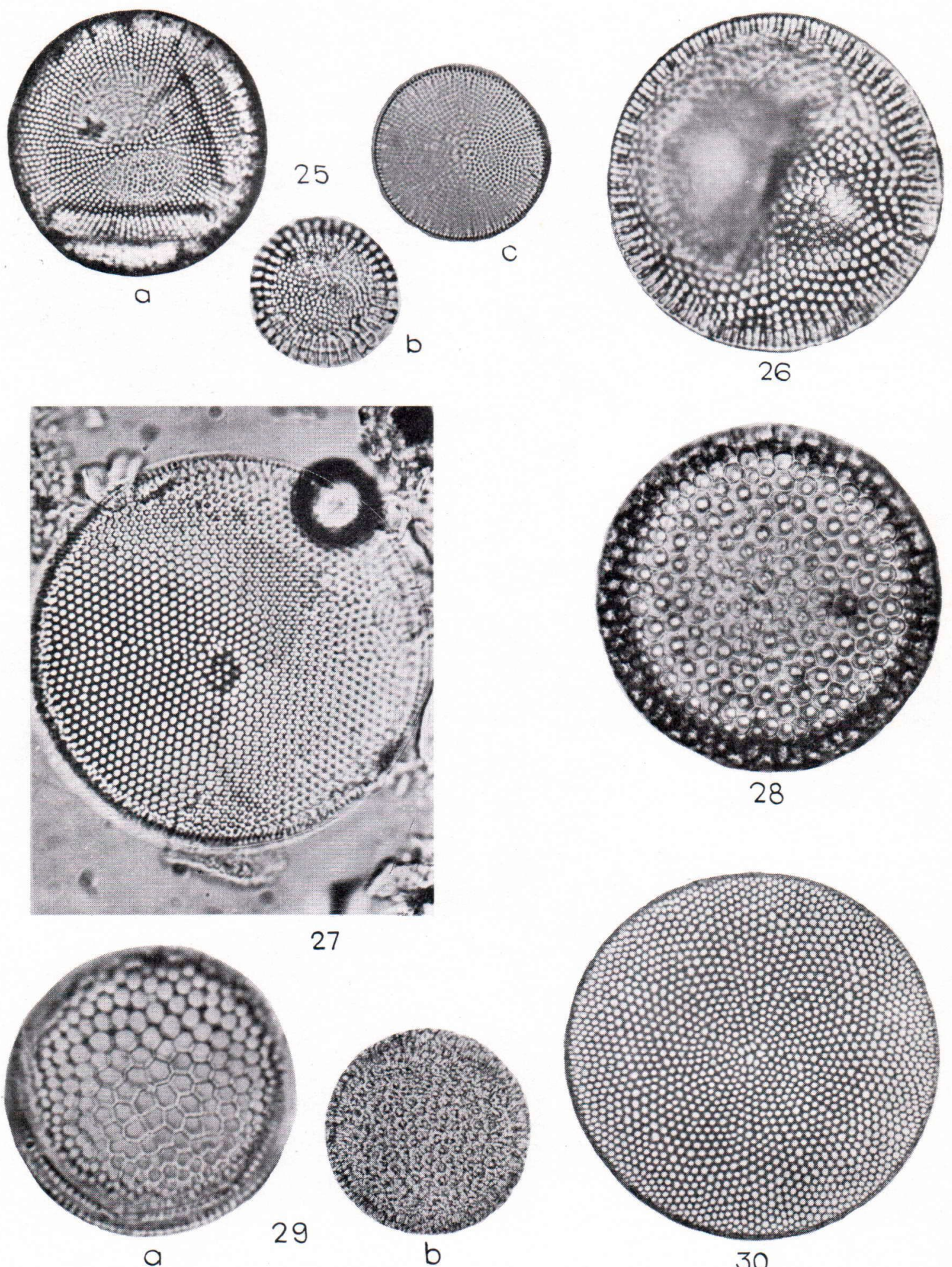

27
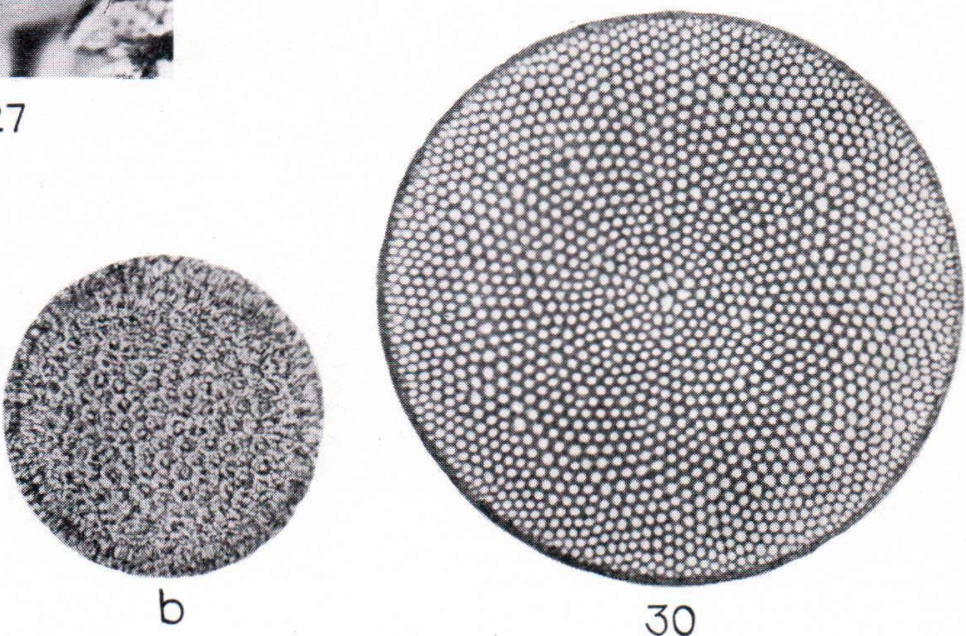

Fig. 25. a-c. Coscinodiscus lacustris, 26. C. l. var. septentrionalis, 27. C. lineatus var. irregularis, 28. C. marginatus, 29. a-b. C. nodulifer, 30. C. oculus iridis. 
TA FEL V
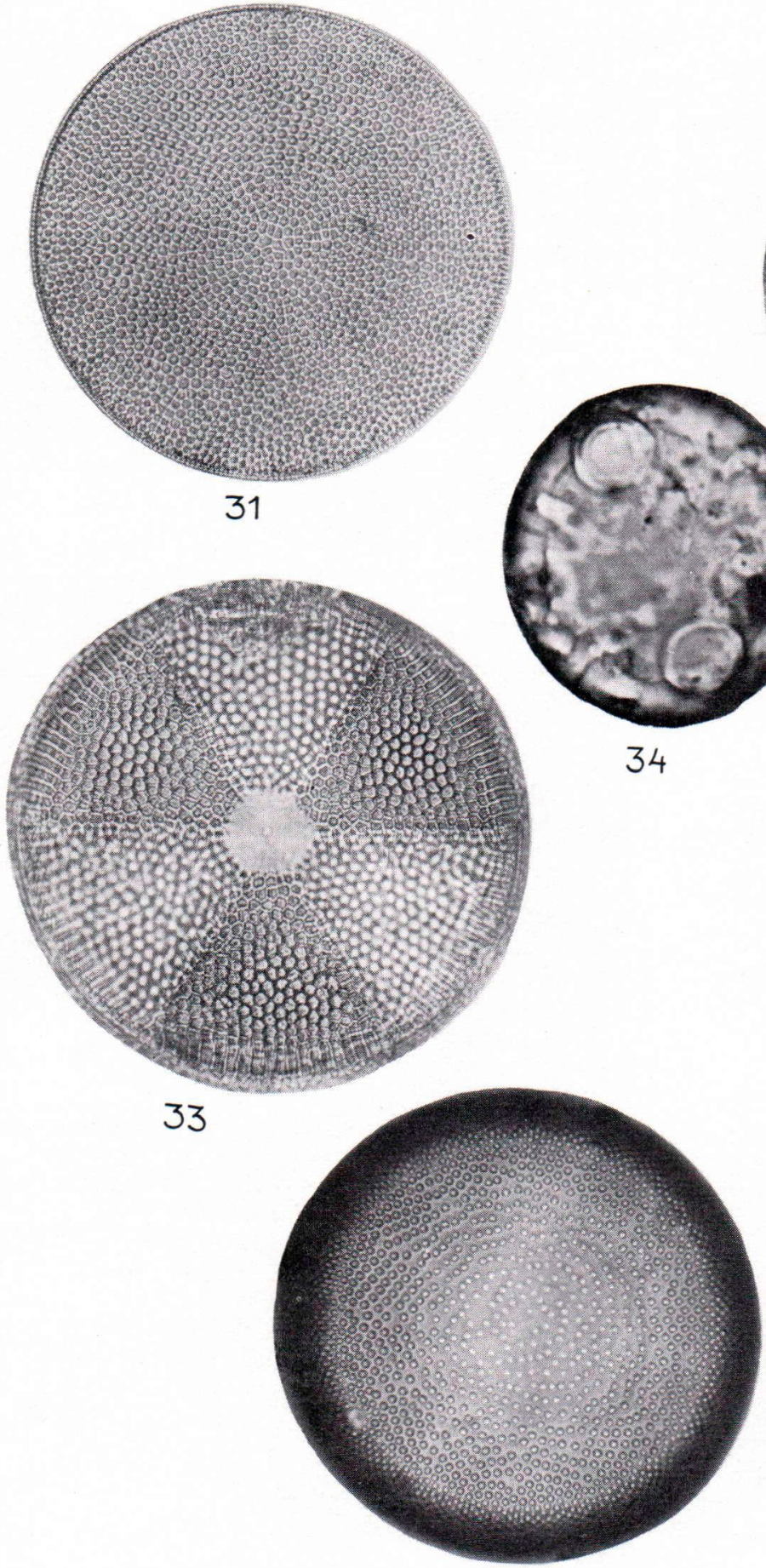

37

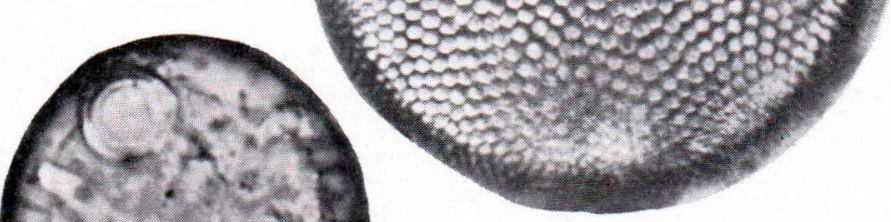

32

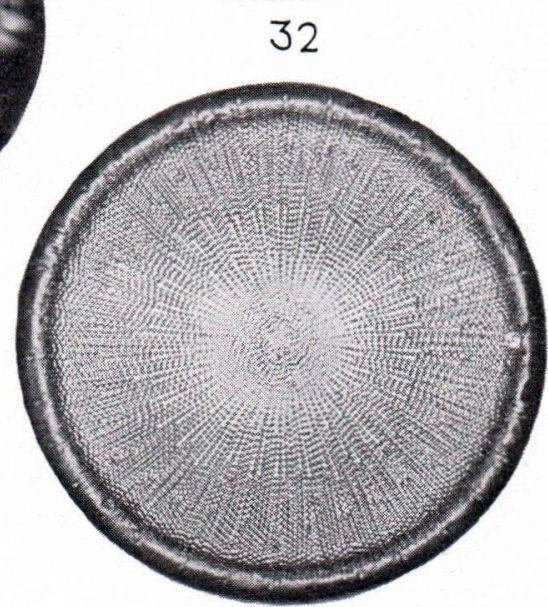

35

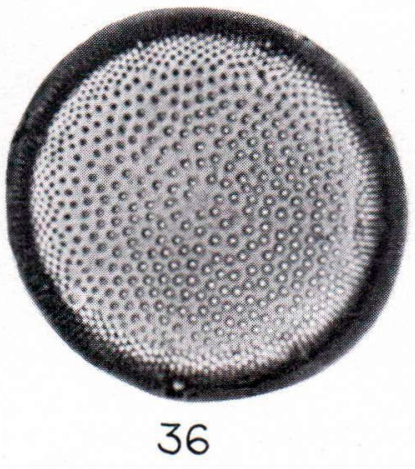

Fig. 31. Coscinodiscus perforatus var. cellulosa, 32. C. radiatus, 33. Actinoptychus undulatus, 34. Auliscus caelatus, 35. Actinocyclus ehrenbergi, 36. A. e. var. crassa, 37. A. e. var. ralfsii. 
TAFEL VI
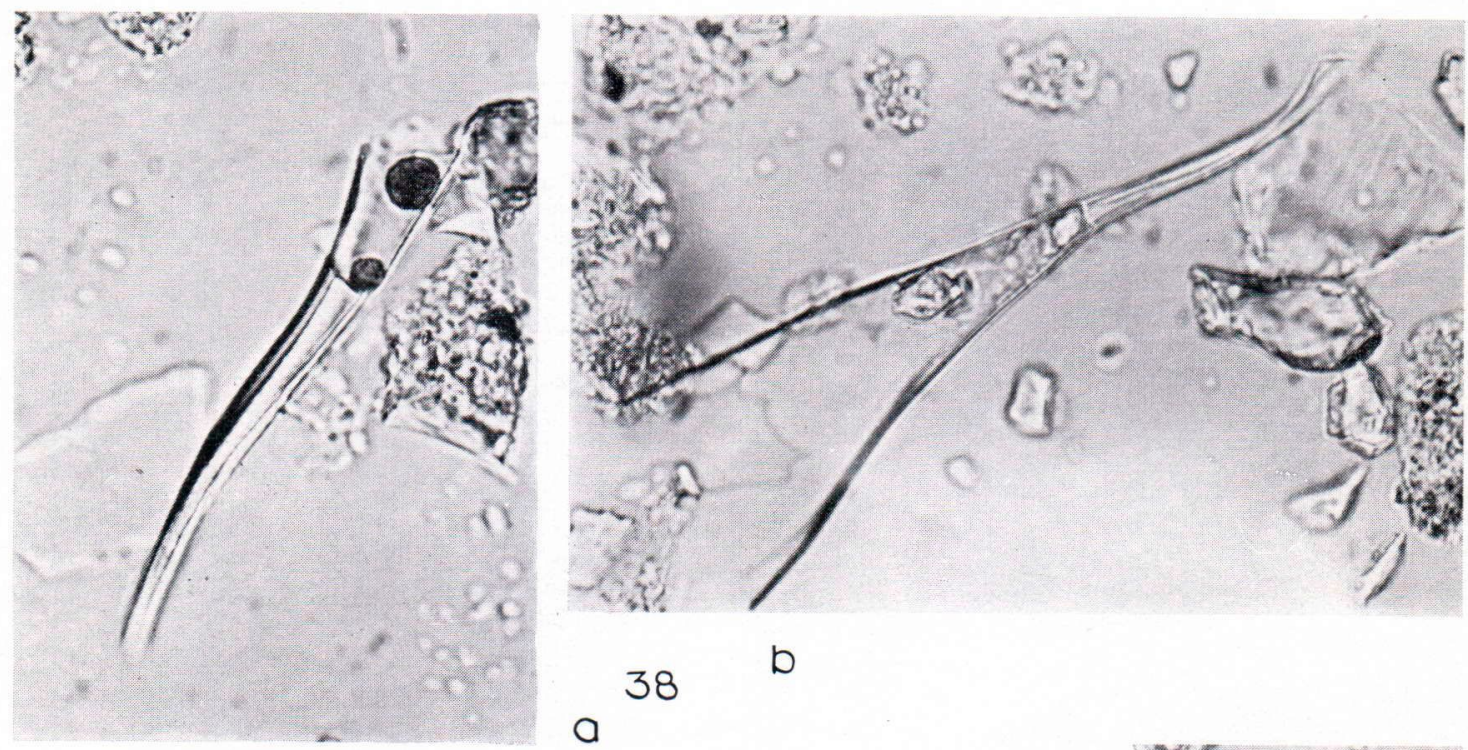

38
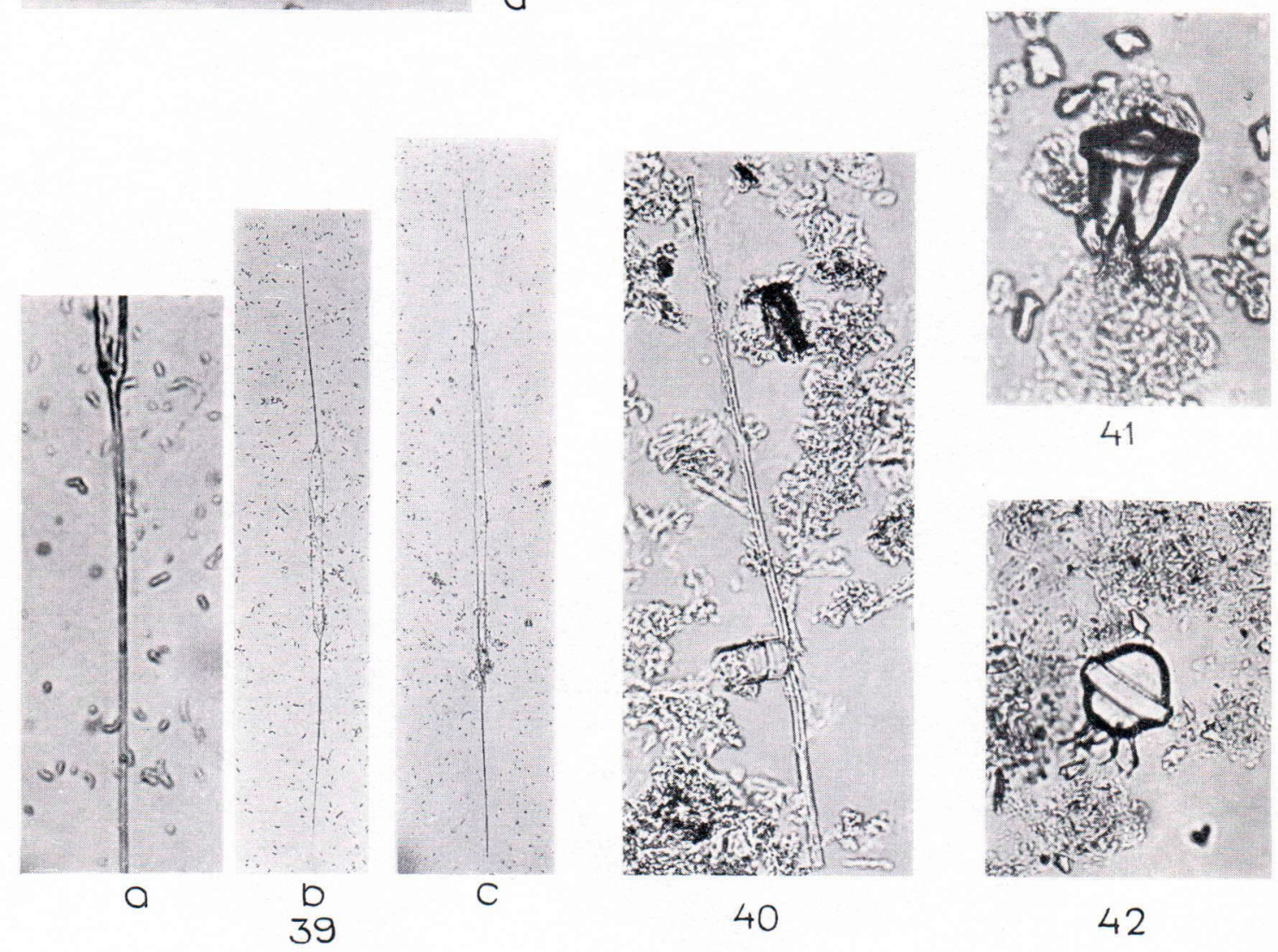

Fig. 38. a-b. Rbizosolenia calcar avis, Fragm., 39. a-c. R. longiseta, 40. Chaetoceros danicus, 41. C. mitra, Dauerspore, 42. C. subsecundus, Dauerspore. 
TAFEL VII
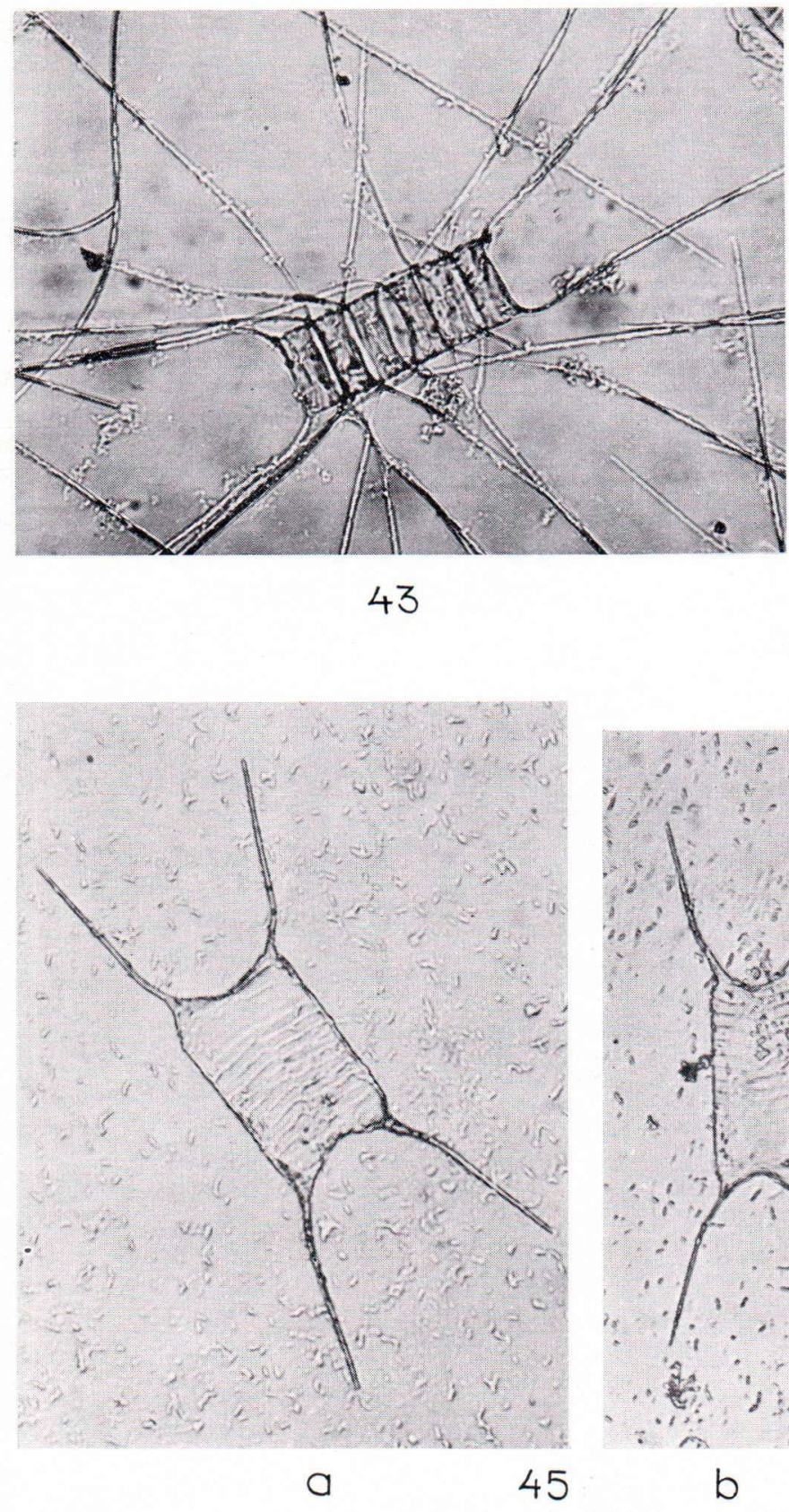

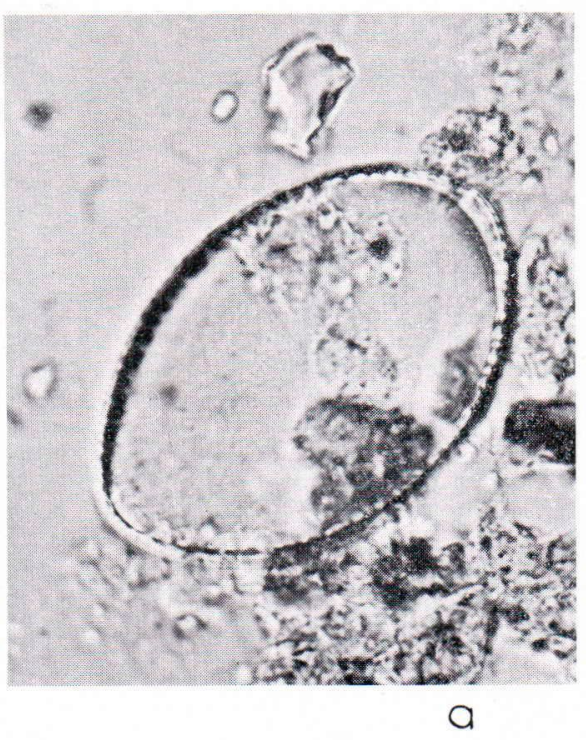

44

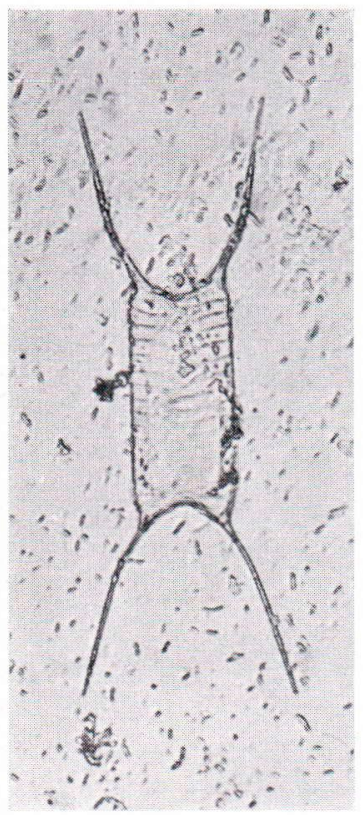

b

Fig. 43. Chaetoceros wighami, 44. a-b. C. sp., Valvarebenen, 45. a-b. Attheya zachariasi. Meistens etwa 1000 -fache Vergrösserung. Photo E. Halme. 


\section{ERGÄNZENDE SCHRIFTEN}

Aario, L. (1932) Planzentopographische und paläogeographische Mooruntersuchungen in N-Satakunta, Fennia 55. 1; Comm. Inat. Forest. Fenniae 17, 1.

Aario, R. (1965b) Die quartäre Schichtenfolge am Flösskanal von Kimola, Südfinnland. Ann. Acad. Sci. Fennica Ser. A. III Geol. -Geogr. 86.

Backman, A. L. \& Astrid Cleve-Euler (1922) Die fossile Diatomeenflora in Österbotten. Acta Forestalia Fennica 22, s. $1-71$.

Brander, G. (1941) Neue Beiträge zur Kenntnis der interglazialen Bildungen in Finnland. C. R. Soc. géol. Finlande 15.

Brander, G \& T. Brenner (1935) Fredriksbergsmossen. Fennia 57.

GARDEMEISTER, R. (1968) Interglasiaalista saviainesta Somerolla? Geologi Nr. 4.

Hendey, Ingmar (1937) The plankton diatoms of the southern seas. Discovery Reports. Vol. 16, s. 151364. Cambridge.

- (1964) An Introductory account of the smaller algae of British coastal waters. Part V, Bacillariophyceae (Diatoms). Fishery Investigations, Ser. 4. London. s. $1-317$.

Huber-Pestalozzi, G. (1942) Das Phytoplankton des Süsswassers 2. Teil. Diatomeen. Die Binnengewässer Bd. XVI, Teil. 2. Stuttgart.
JäRNEFELT, H. (1934) Zur Limnologie einiger Gewässer Finnlands X. Ann. Zool. Bot. Fennica »Vanamo» Tom. 14. s. $1-44$.

KILPI, S. (1937) Das Sotkamo-Gebiet in spätglazialer Zeit. Bull. Comm. géol. Finlande 177.

Levander, K. M. (1904) Zur Kenntnis der Rhizosolenien Finnlands. Medd. Pro Fauna et Flora Fennica 39. s. $112-117$.

- Till kännedom om planktonbeskaffenheten i Helsingfors inre hamnar. Medd. Pro Fauna et Flora Fennica 39 , s. $26-36$.

Lindeber G, H. (1910) Resultaten av de phytopaleontologiska undersökningarna inom Lojo härad. Finska Mosskulturfören. årsbok. 1910, Häft. 2. s. 318-347.

Meriläinen, J. (1967) The diatom flora and the hydrogen ion concentration of the water. Ann. Bot. Fennici $4,51-58$.

Purasjoki, K. J. (1947) Plankton gesammelt in den Jahren 1899-1910 an den Küsten Finnlands. Finnländische Hydrogr.-Biol. Untersuch. Nr. 11, s. 1-40.

Wuorentaus, Y. (1913) Tietoja Pohjanlahden rannikkoplanktonista. Medd. Pro Fauna et Flora Fennica 39, s. $15-25$.

Manuskript eingegangen am 8. April 1968.

Anschrift: Prof. Karl Mölder Kelohongantie 2 C 21 